\title{
əFrequency-Dependent Estimation of Effective Spatial Degrees of Freedom
}

\author{
TORBEN KUnZ ${ }^{\mathrm{a}}$ AND THOMAS LAEPPLE ${ }^{\mathrm{a}, \mathrm{b}}$ \\ a Alfred-Wegener-Institut Helmholtz-Zentrum für Polar- und Meeresforschung, Research Unit Potsdam, Potsdam, Germany \\ ${ }^{\mathrm{b}}$ MARUM-Center for Marine Environmental Sciences and Faculty of Geosciences, University of Bremen, Bremen, Germany
}

(Manuscript received 1 April 2020, in final form 4 June 2021)

\begin{abstract}
Climate variability occurs over wide ranges of spatial and temporal scales. It exhibits a complex spatial covariance structure, which depends on geographic location (e.g., tropics vs extratropics) and also consists of a superposition of (i) components with gradually decaying positive correlation functions and (ii) teleconnections that often involve anticorrelations. In addition, there are indications that the spatial covariance structure depends on frequency. Thus, a comprehensive assessment of the spatiotemporal covariance structure of climate variability would require an extensive set of statistical diagnostics. Therefore, it is often desirable to characterize the covariance structure by a simple summarizing metric that is easy to compute from datasets. Such summarizing metrics are useful, for example, in the context of comparisons between climate models or between models and observations. Here we introduce a frequency-dependent version of a simple measure of the effective spatial degrees of freedom. The measure is based on the temporal variance of the global average of some climate variable, and its novel aspect consists in its frequency dependence. We also provide a clear geometric interpretation of the measure. Its easy applicability is demonstrated using near-surface temperature and precipitation fields obtained from a paleoclimate model simulation. This application reveals a distinct scaling behavior of the spatial degrees of freedom as a function of frequency, ranging from monthly to millennial scales.
\end{abstract}

KEYWORDS: Atmospheric circulation; Large-scale motions; Climate variability; Paleoclimate; Spectral analysis/models/ distribution

\section{Introduction}

Quantification of climate variability requires, from a statistical point of view, the estimation of its full spatiotemporal covariance function. Given the nonstationarity of the variability, this is not only a function of spatial distance in either direction and of time lag; it also depends on space and time. Even for a two-dimensional spatial domain (e.g., for global temperature fields), this yields already a six-dimensional covariance function. Therefore, it is often desirable to define a simple metric that concisely summarizes the covariance structurewhether it be for climate model intercomparison or validation, for the formulation of stochastic forecasting models (Lovejoy et al. 2018), or for assigning a representative spatiotemporal scale to an observational data point (Smith et al. 1994), as needed for interpolation of spatially incomplete fields (Cowtan and Way 2014) or for data assimilation into paleoclimate models (Hakim et al. 2016).

To characterize the spatial structure, a common approach is to define a measure of the effective spatial degrees of freedom (ESDOF), to be interpreted as the effective number of independent spatial samples. Thus, the size of the entire spatial domain divided by this number equals the effective spatial extent of the associated fluctuations. Inherent to any ESDOF measure is a spatial averaging operator, implicitly removing any information on spatial nonstationarity. Effectively, the entire spatial correlation structure is condensed into a single

¿ Denotes content that is immediately available upon publication as open access.

Corresponding author: Torben Kunz, torben.kunz@awi.de number. A variety of ESDOF measures of different complexity have been presented in the literature (Livezey and Chen 1983; Smith et al. 1994; Jones et al. 1997; Wang and Shen 1999; Bretherton et al. 1999, and references therein).

To also consider the temporal structure, the variability may be filtered in the time domain before application of an ESDOF measure. Jones et al. (1997), for example, apply their ESDOF measure to annual mean and to decadal mean temperature fields, and find a reduction of the ESDOFs by roughly a factor of 2 toward decadal scales, both for observations and models. In qualitative agreement with this are the results of North et al. (2011) and Rypdal et al. (2015), who demonstrate that in a simple diffusive energy balance model (EBM) the decorrelation length scale increases toward lower frequencies, which is consistent with fewer ESDOFs at longer time scales. The latter study confirms this result also for a climate model simulation over a range of frequencies from annual up to centennial time scales. These findings motivate us to formulate a frequency-dependent ESDOF measure as a simple summarizing metric of climate variability.

Section 2 illustrates the frequency dependence of the spatial correlation structure, using a paleoclimate model simulation. This further motivates the formulation of a frequency-dependent ESDOF measure to be introduced in section 3. Application examples are presented in section 4 , followed by a discussion of the measure in section 5. The conclusions and potential future applications are itemized in section 6 .

\section{Frequency dependence of the spatial correlation structure}

This section illustrates the fact that the spatial covariance structure of climate variability may indeed exhibit a notable frequency dependence. This is demonstrated, in particular, 

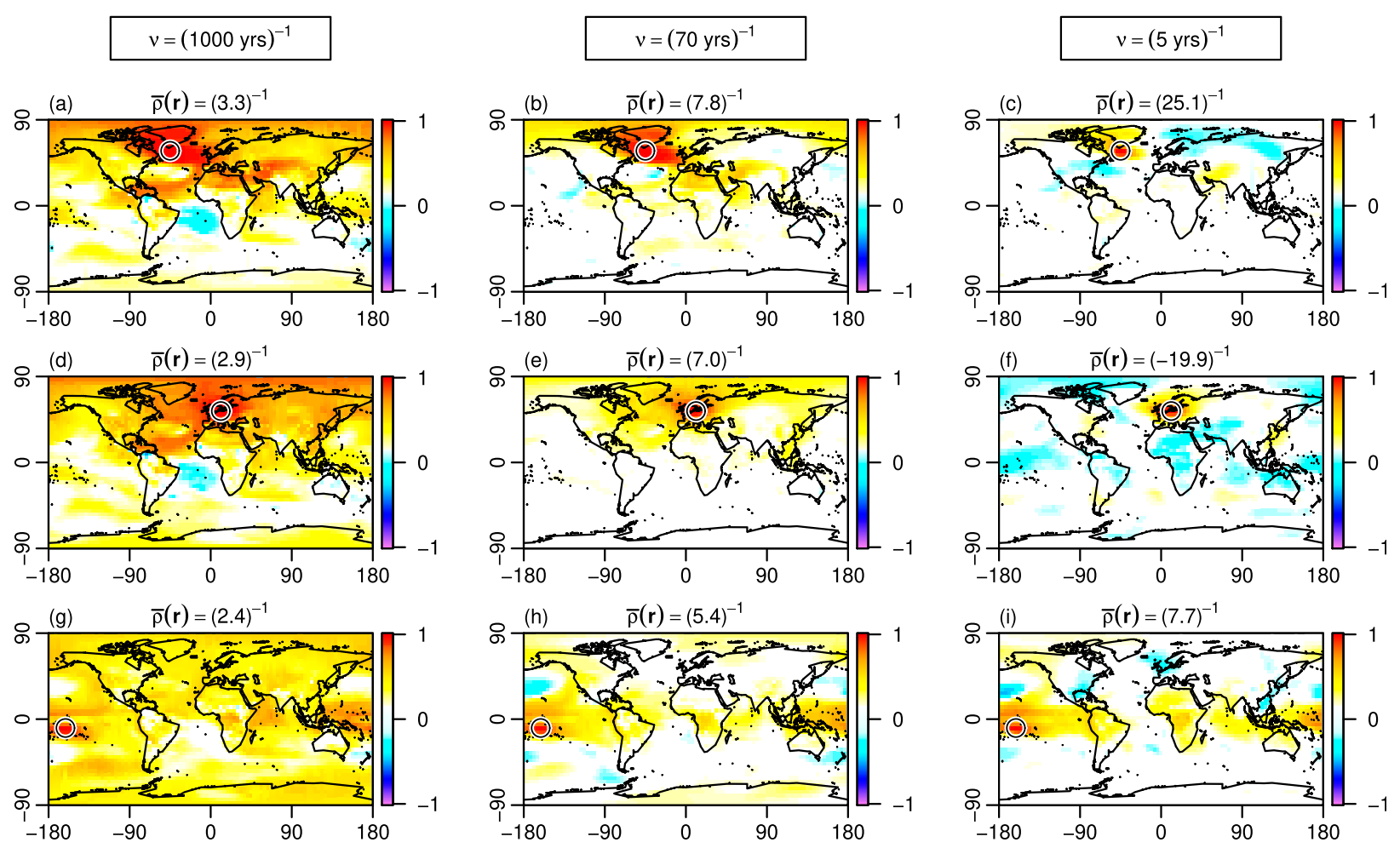

FIG. 1. Spatial correlation function $\rho\left(\mathbf{r}, \mathbf{r}^{\prime}\right)$ of 2-m temperature, obtained from the TraCE-21ka paleoclimate model simulation, using 7000 years until $1890 \mathrm{CE}$. Colors indicate the correlation between the reference location $\mathbf{r}$, marked by the black circle, and any other location $\mathbf{r}^{\prime}$ on the globe. Reference locations $\mathbf{r}$ are at (top) $57.5^{\circ} \mathrm{N}, 45.0^{\circ} \mathrm{W}$, (middle) $53.8^{\circ} \mathrm{N}, 11.3^{\circ} \mathrm{E}$, and (bottom) $9.3^{\circ} \mathrm{S}, 161.3^{\circ} \mathrm{W}$. Correlations are shown for three different frequency bands centered at (left) $\nu=(1000 \mathrm{yr})^{-1}$, (middle) $\nu=(70 \mathrm{yr})^{-1}$, and (right) $\nu=(5 \mathrm{yr})^{-1}$ (correlation estimates are based on $\kappa=180$ degrees of freedom). The global average (with respect to $\left.\mathbf{r}^{\prime}\right)$ of the correlation function, $\bar{\rho}(\mathbf{r})$, is specified above each panel, which can be interpreted as the fraction of the Earth's surface covered by the effective spatial extent of the fluctuations associated with $\mathbf{r}$. Thus, the reciprocal of this average specifies how many times the effective spatial extent fits onto the globe.

for near-surface temperature variability generated by a paleoclimate model simulation, which allows us to investigate a wide range of frequencies from monthly to millennial time scales.

Specifically, we use monthly mean 2-m temperature fields from the Transient Climate of the Last 21,000 Years (TraCE$21 \mathrm{ka}$ ) coupled general circulation model simulation (Liu et al. 2009; He 2011) with T31 atmospheric horizontal resolution. We restrict our analysis to the preindustrial Holocene by selecting the last 7000 years under preindustrial conditions (i.e., up to $1890 \mathrm{CE}$ ) of the simulation, including only natural (i.e., nonanthropogenic) external forcings [for a detailed description of the forcings, see He (2011)]. Finally, the obtained time series are linearly detrended at each grid point.

From these data we compute the spatial correlation function, defined as $\rho\left(\mathbf{r}, \mathbf{r}^{\prime}\right)=R\left(\mathbf{r}, \mathbf{r}^{\prime}\right) /\left[\sigma(\mathbf{r}) \sigma\left(\mathbf{r}^{\prime}\right)\right]$, where $R\left(\mathbf{r}, \mathbf{r}^{\prime}\right)$ is the covariance between a reference location $\mathbf{r}$ and any other location $\mathbf{r}^{\prime}$ on the globe, and $\sigma^{2}(\mathbf{r})=R(\mathbf{r}, \mathbf{r})$ is the local variance. To demonstrate frequency dependence, correlations are estimated from the data within narrow frequency bands centered at various frequencies $\nu$. These frequency-dependent correlation estimates are based on the smoothed and normalized cospectral density, as detailed in section $3 \mathrm{~d}$, including only information on simultaneous correlation. Figure 1 shows the spatial correlation function for three different frequencies, which correspond to millennial, multidecadal, and interannual time scales, and for three selected reference locations. These are located over the northern North Atlantic, over central Europe, and over the western tropical Pacific.

It turns out that the area that is positively correlated with the reference location (red and yellow colors), which characterizes the spatial extent of the temperature fluctuations, increases with time scale. In addition, the spatial extent varies with latitude, most strikingly at interannual time scales. In this frequency band, the largest positively correlated area is found for the tropical reference location, extending over most of the tropical belt around the globe, in contrast to the much smaller decorrelation length scales found in the extratropics. These findings are consistent with the results of Jones et al. (1997, see their Figs. 3 and 4), comparing decorrelation length scales at interannual and decadal time scales, obtained from both observations and general circulation models. Furthermore, for some reference locations, most clearly for the tropical one at interannual and multidecadal time scales, the spatial correlation function exhibits a large degree of anisotropy, which is also in agreement with the analysis of model temperature data by Jones et al. (1997, see their Fig. B1).

To quantify the spatial extent of the temperature fluctuations, Jones et al. (1997) fit an exponential decay function 

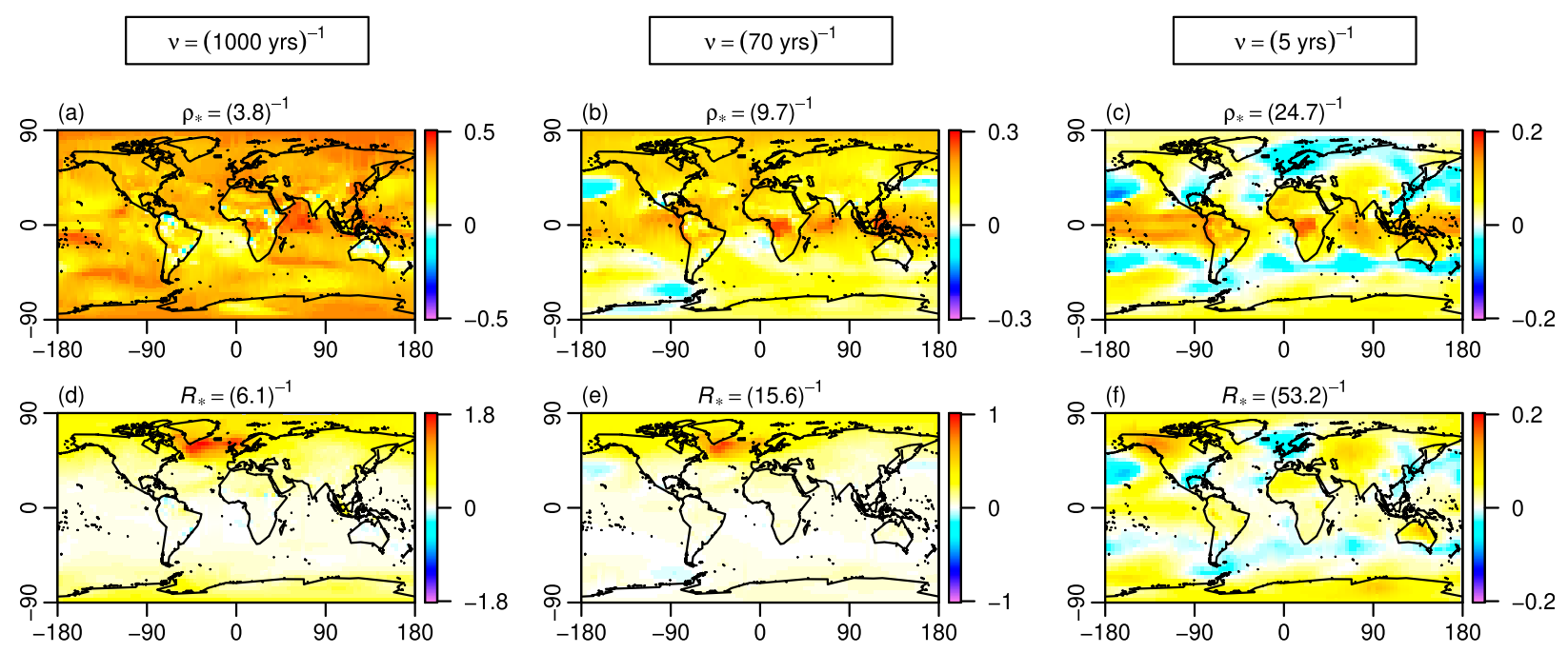

FIG. 2. (top) Maps of the global average of the spatial correlation function $\bar{\rho}(\mathbf{r})$ of $2-\mathrm{m}$ temperature; model data and frequency bands (from left to right) as in Fig. 1. The global average of this quantity $\rho_{\star}$ is specified above each panel. (bottom) As in the top row, but for the normalized covariance function, $\bar{R}(\mathbf{r}) / \overline{\sigma^{2}}$, instead of the correlation function. The global average of this quantity $R_{\star}$ is specified above each panel. The reciprocals of $\rho_{\star}$ and $R_{\star}$ are equal to the ESDOF measures $D_{\rho}$ and $D_{R}$, respectively, to be defined in section 3 a.

to the estimated correlation as a function of spatial distance. An alternative measure of the spatial extent is the global average (or integral) of the spatial correlation function. An advantage of this measure is its insensitivity to anisotropic structures. In Fig. 1, the global average (with respect to $\mathbf{r}^{\prime}$ ) of the spatial correlation function for a particular reference location $\mathbf{r}$, denoted by $\bar{\rho}(\mathbf{r})$, is specified at the top of each panel. This average can be interpreted as the fraction of Earth's surface covered by the effective spatial extent of the fluctuations associated with $\mathbf{r}{ }^{1}$ The obtained values reconfirm the above findings regarding the dependence of the spatial extent on frequency and location. For the reference location over the northern North Atlantic (top row), for example, $\bar{\rho}(\mathbf{r})$ ranges from about $1 / 3$ at millennial time scales to $1 / 8$ at multidecadal and $1 / 25$ at interannual time scales. Thus, the associated fluctuations, at these three different time scales, have an effective spatial extent that fits 3,8 , and 25 times onto the globe, respectively.

In addition to this frequency dependence of the effective spatial extent, Fig. 1 also reveals an increased occurrence of anticorrelations at short time scales. In particular, at interannual time scales (right column), considerable areas are anticorrelated with the reference location (blue colors), preferably in the extratropics. Although some of those negative correlation estimates might not be statistically significant, it suggests that at those time scales and locations the correlation structure is notably influenced by teleconnections, such as the North Atlantic Oscillation, Pacific-North American pattern, or El Niño-Southern Oscillation (ENSO) phenomenon [see, e.g.,

\footnotetext{
${ }^{1}$ This interpretation holds because the average $\bar{\rho}(\mathbf{r})$ would attain the same value if $\mathbf{r}$ would be perfectly correlated with that area fraction and fully uncorrelated with the remaining area (keeping the integral of the spatial correlation function constant; see section $3 \mathrm{~b}$ for further interpretation).
}

Wallace and Gutzler (1981) or Feldstein and Franzke (2017) for an overview of atmospheric teleconnections]. In some cases this may even lead to a negative global average of the correlation function, an example of which is given by the reference location over central Europe at interannual time scales (Fig. 1f). In these cases the global average of the spatial correlation function has a different interpretation, as to be explained in the next section in the context of the ESDOF measures.

Whereas in Fig. 1 maps of $\rho\left(\mathbf{r}, \mathbf{r}^{\prime}\right)$ are shown, with fixed $\mathbf{r}$ and varying $\mathbf{r}^{\prime}$, Fig. 2 (top row) shows maps of $\bar{\rho}(\mathbf{r})$, with varying $\mathbf{r}$, again for the same three frequencies as before. These results confirm the previous findings, namely that (i) the effective spatial extent increases with time scale, (ii) it increases toward the tropics, particularly at interannual time scales, and (iii) anticorrelations occur preferably at these shorter time scales and in the extratropics, suggesting an involvement of teleconnections. At the top of each panel in Fig. 2 (top row) the global average of $\bar{\rho}(\mathbf{r})$ is specified, defined as

$$
\rho_{\star}=\overline{\overline{\left[\frac{R\left(\mathbf{r}, \mathbf{r}^{\prime}\right)}{\sigma(\mathbf{r}) \sigma\left(\mathbf{r}^{\prime}\right)}\right]}},
$$

where the first averaging operator applies to $\mathbf{r}^{\prime}$ and the second one to r. From the specified values, the fluctuations at millennial, multidecadal and interannual time scales have an average spatial extent that fits roughly 4, 10, and 25 times onto the globe, respectively.

According to (1), the average spatial extent $\rho_{\star}$ is obtained by first normalizing the spatial covariance function $R\left(\mathbf{r}, \mathbf{r}^{\prime}\right)$ in amplitude, and then averaging it in space. An alternative measure of the average spatial extent can be obtained by reversing the order of operations, defined as

$$
R_{\star}=\frac{\overline{\overline{R\left(\mathbf{r}, \mathbf{r}^{\prime}\right)}}}{\overline{\sigma^{2}}},
$$


where in the numerator the first averaging operator applies again to $\mathbf{r}^{\prime}$ and the second one to $\mathbf{r}$, and $\overline{\sigma^{2}}$ in the denominator is the global average of the local variance $\sigma^{2}(\mathbf{r})$. Because $R \star$ is based on the spatial distribution of covariance, rather than correlation, it represents an average weighted by the local variance, in contrast to $\rho_{\star}$. Thus, the correlation structure in regions with large local variance has a greater impact on the value of $R_{\star}$ than the structure in regions with small variance.

The effect of this variance weighting can be seen in Fig. 2 (bottom row), showing maps of $\bar{R}(\mathbf{r}) / \overline{\sigma^{2}}$, which also has the interpretation of an effective spatial extent; and where $\bar{R}(\mathbf{r})$ is the global average (with respect to $\left.\mathbf{r}^{\prime}\right)$ of $R\left(\mathbf{r}, \mathbf{r}^{\prime}\right)$. Smallest values are preferably found in the tropics, particularly at millennial to multidecadal time scales, and largest values in the northern extratropics, which is consistent with typical spatial variance patterns of near-surface temperature. The global average of this effective spatial extent is equal to $R_{\star}$, the value of which is specified at the top of each panel. It turns out that $R_{\star}$ also increases with time scale, but values are smaller than those of $\rho_{\star}$ (top row), consistent with the above finding that the temperature fluctuations in the extratropics have a smaller effective spatial extent and are more affected by anticorrelations due to teleconnections. From the specified values of $R_{\star}$, the variance-weighted fluctuations at millennial, multidecadal, and interannual time scales have an average spatial extent that fits roughly 6,16 , and 53 times onto the globe, respectively. It is this type of information that we intend to characterize and quantify with a frequency-dependent ESDOF measure, to be defined in the next section.

\section{Effective spatial degrees of freedom}

In this section a simple ESDOF measure is defined and its interpretation is provided in terms of idealized spatial fields. Subsequently, estimators of slightly different variants of the measure are presented and their basic properties are specified. Finally, the measures are equipped with an explicit dependence on frequency, allowing for the estimation of ESDOFfrequency spectra, which can then serve to infer the basic space-time scaling behavior of climate variability.

\section{a. Definition}

Various ESDOF definitions have been proposed in the literature, each of which has its specific interpretation. Accordingly, a number of ESDOF measures of different complexity have been presented, depending on the purpose of application. These range from simple variance ratios computed directly from the time-varying spatial fields [as used by, e.g., Smith et al. (1994) and Jones et al. (1997)] to ratios of highermoment statistics, sometimes involving principal component analysis, and moment matching approaches [see Wang and Shen (1999) and Bretherton et al. (1999), and references therein, for an overview]. Here we are adopting one of the simplest ESDOF definitions that is based on a variance ratio and that is directly related to the average spatial extent of the fluctuations discussed in the previous section.

For a precise definition, consider a zero-mean random field $X(\mathbf{r})$, defined on a continuous spatial domain $\mathcal{S}$ of finite size $A$, where $\mathbf{r} \in \mathcal{S}$ is a position vector and $A=\int_{\mathcal{S}} d \mathcal{A}(\mathbf{r})$, with the Lebesgue measure $\mathcal{A}$. Although, in general, the domain $\mathcal{S}$ could be of any dimension, in the following we assume the domain be two-dimensional and spherical (unless otherwise noted), in which case $A$ is an area and the differential $d \mathcal{A}(\mathbf{r})$ represents an area increment. For example, $X(\mathbf{r})$ could be a global temperature field and $A$ the surface area of Earth. The spatial covariance function can then be expressed as $R\left(\mathbf{r}, \mathbf{r}^{\prime}\right)=\left\langle X(\mathbf{r}) X\left(\mathbf{r}^{\prime}\right)\right\rangle$, where $\langle\cdot\rangle$ denotes the expected value operator with respect to the realization of the random field, and the spatial averaging operator $\overline{(\cdot)}$ can be defined as $\bar{x}=A^{-1} \int_{\mathcal{S}} x(\mathbf{r}) d \mathcal{A}(\mathbf{r})$ for any spatial field $x(\mathbf{r})$.

As illustrated in the previous section, the averaged and normalized spatial covariance function $R_{\star}$, defined by (2), represents the average spatial extent of the fluctuations of the field, as a fraction of the size $A$ of the domain. Thus, its reciprocal can be interpreted as the effective number of independent spatial samples. Accordingly, a simple ESDOF measure can be defined as

$$
\begin{aligned}
D_{R} & =1 / R_{\star} \\
& =\overline{\sigma^{2}} / \sigma_{0}^{2},
\end{aligned}
$$

where $\sigma_{0}^{2}$ denotes the variance of the spatial average $\bar{X}$, and the second step follows from (2) and from the fact that $\sigma_{0}^{2}=\left\langle\bar{X}^{2}\right\rangle=$ $A^{-2} \int_{\mathcal{S}} \int_{\mathcal{S}}\left\langle X(\mathbf{r}) X\left(\mathbf{r}^{\prime}\right)\right\rangle d \mathcal{A}\left(\mathbf{r}^{\prime}\right) d \mathcal{A}(\mathbf{r})=A^{-1} \int_{\mathcal{S}} \bar{R}(\mathbf{r}) d \mathcal{A}(\mathbf{r})=\overline{\bar{R}} \cdot{ }^{2}$ Thus, the ESDOF measure $D_{R}$ is given by the ratio of the spatial average of the local variance to the variance of the spatial average, and it is identical to the measure of Smith et al. (1994) [see their Eq. (7)] and of Jones et al. (1997) [see their Eq. (10)]. Alternatively, an ESDOF measure can be defined based on the averaged spatial correlation function $\rho_{\star}$, defined by (1), as

$$
D_{\rho}=1 / \rho_{\star}
$$

Note that for random fields with stationary variance, it follows from (1) and (2) that $R_{\star}=\rho_{\star}$ and, thus, $D_{R}=D_{\rho}$, where here and in the following stationarity refers to the spatial dimension (unless otherwise noted). For fields with nonstationary variance, the measure $D_{R}$ depends on the spatial distribution of the local variance, as discussed in the previous section in the context of $R_{\star}$. In practical applications to nonstationary fields, the measure $D_{\rho}$ can be obtained by standardizing the field at each location, as detailed in section $3 \mathrm{c}$.

Whereas the above ESDOF measures are based on the integral of the spatial covariance or correlation function, it can sometimes be useful to construct alternative ESDOF measures that are based on other aspects of the spatial correlation structure-for example, the $e$-folding length scale of the correlation function. In particular, for spherical domains, where

\footnotetext{
${ }^{2}$ Note that, for a discrete spatial field, the two averages involved in $\overline{\bar{R}}$ correspond to the (area weighted) sums over the rows and over the columns of the covariance matrix.
} 
the distance between two locations $\mathbf{r}$ and $\mathbf{r}^{\prime}$ can be expressed in terms of their angular distance $\theta$, one can define the radial covariance function $R(\theta, \mathbf{r})=\overline{R\left(\mathbf{r}, \mathbf{r}^{\prime}\right)^{(\theta)}}$ for a particular reference location $\mathbf{r}$. Here, the operator $\overline{(\cdot)^{(\theta)}}$ denotes the spatial average over all $\mathbf{r}^{\prime}$ with the same angular distance $\theta$ relative to $\mathbf{r}$. With this, one can then define the normalized average radial covariance function,

$$
R_{\star}(\theta)=\overline{R(\theta, \mathbf{r})} / \overline{\sigma^{2}}
$$

where the spatial average applies to $\mathbf{r}$ as previously defined. Likewise one can define the radial correlation function $\rho(\theta, \mathbf{r})=\overline{\rho\left(\mathbf{r}, \mathbf{r}^{\prime}\right)^{(\theta)}}$ and, based thereon, the average radial correlation function,

$$
\rho_{\star}(\theta)=\overline{\rho(\theta, \mathbf{r})} .
$$

Note that $R_{\star}(0)=\rho_{\star}(0)=1$ and that $R_{\star}(\theta), \rho_{\star}(\theta) \leq 1$ for $\theta>0$.

If, additionally, the spatial domain covers the entire sphere, such that the domain is cyclic in each direction and $\theta \in[0, \pi]$, then the radial functions $R_{\star}(\theta)$ and $\rho_{\star}(\theta)$ are directly related to the above ESDOF measures $D_{R}$ and $D_{\rho}$, respectively. Specifically, by defining the global, area-weighted mean of any radial function $x(\theta)$ over the interval $[0, \pi]$ as $M[x(\theta)]=\left[\int_{0}^{\pi} x(\theta) \sin \theta d \theta\right] / 2$, we can write $R_{\star}=M\left[R_{\star}(\theta)\right]$ and $\rho_{\star}=M\left[\rho_{\star}(\theta)\right]$, from which the ESDOF measures $D_{R}$ and $D_{\rho}$ are readily obtained, according to (3) and (5), respectively. Now, in order to construct an alternative ESDOF measure, one can fit a parametric correlation function, for example, an exponential decay function, to either $R_{\star}(\theta)$ or $\rho_{\star}(\theta)$. If we denote the fitted parametric radial functions by $R_{\star, \text { fit }}(\theta)$ and $\rho_{\star, \text { fit }}(\theta)$, respectively, we can define the ESDOF measures

$$
D_{R, \mathrm{fit}}=1 / M\left[R_{\star, \mathrm{fit}}(\theta)\right]
$$

and

$$
D_{\rho, \mathrm{fit}}=1 / M\left[\rho_{\star, \mathrm{fit}}(\theta)\right],
$$

where the latter one corresponds to the second ESDOF measure of Jones et al. (1997) [see their Eq. (14)] if $\rho_{\star \text {,fit }}(\theta)$ is an exponential decay function fit to $\rho_{\star}(\theta)$ over the entire interval $[0, \pi]$. Note that for random fields with stationary variance, $R_{\star}(\theta)=\rho_{\star}(\theta)$ and, thus, $D_{R, \text { fit }}=D_{\rho, \text { fit }}$.

In the present study, we use $D_{R}$ as our main ESDOF measure. We compare $D_{R}$ to $D_{\rho}$ to demonstrate possible effects of data standardization, and we also compare $D_{R}$ to $D_{R \text {,fit }}$ to illustrate how various aspects of the radial correlation structure may affect the measure $D_{R}$ in different ways. Furthermore, because $D_{R}$ is identical to the first ESDOF measure of Jones et al. (1997) [see their Eq. (10)], we can compare estimates of this measure between the two studies. Finally, one can loosely compare estimates of $D_{R \text {,fit }}$ to the second ESDOF measure of Jones et al. (1997) [see their Eq. (14)] because it corresponds to the closely related measure $D_{\rho \text {,fit }}$. An overview of the various measures and their relation to those of Jones et al. (1997) is provided by Table 1 .
TABLE 1. List of ESDOF measures defined in the present study and their correspondence to those previously defined by Jones et al. (1997).

\begin{tabular}{lccc}
\hline \hline Symbol & Defined by & $\begin{array}{c}\text { Used in } \\
\text { this study }\end{array}$ & $\begin{array}{c}\text { Corresponds to } \\
\text { Jones et al. (1997) }\end{array}$ \\
\hline$D_{R}$ & Eq. (4) & Yes & Their Eq. (10) \\
$D_{\rho}$ & Eq. (5) & Yes & - \\
$D_{R, \text { fit }}$ & Eq. (8) & Yes & - \\
$D_{\rho \text {,fit }}$ & Eq. (9) & No & Their Eq. (14) \\
\hline
\end{tabular}

\section{b. Interpretation}

\section{1) Sensitivity to THE CORRELAtion STRuCture}

A few idealized example cases are presented to illustrate the interpretation of the ESDOF measures $D_{R}$ and $D_{\rho}$ for different spatial correlation structures. Since only the correlation structure varies between these cases, we can consider fields with stationary variance, such that $D_{R}=D_{\rho}$, and simply write $D$ for the ESDOF measure. We distinguish between cases without and cases with anticorrelations.

As an example without anticorrelations and with stationary correlation function, consider the idealized case of a random field defined on the entire sphere, and which has a rotationally symmetric correlation function, falling off from one at the reference location to zero at the opposite point on the sphere, according to a cosine bell-shaped structure [i.e., according to $\left.\cos ^{2}(\theta / 2)\right]$, in which case $\rho_{\star}=1 / 2$. Thus, the fluctuations of the random field have an average spatial extent of $A / 2$, which fits $D=2$ times onto the sphere. As an example with nonstationary correlation function, consider the idealized case of coherent but mutually uncorrelated hemispheres, that is, with a correlation of one for all pairs of locations within a hemisphere, but with zero interhemispheric correlation. Again $\rho_{\star}=1 / 2$, because each hemisphere has a spatial extent of $A / 2$. Likewise, one could divide the entire domain $\mathcal{S}$ into $N_{\text {box }}$ equally sized and uncorrelated boxes, such that $\rho_{\star}=1 / N_{\text {box }}$ and, thus, $D=N_{\text {box }}$.

In cases without anticorrelations, it can be useful to introduce an associated length scale measure $L$. For spherical domains, it may be defined as the radius of a spherical cap, the area of which covers that fraction of $A$ that is equal to $\rho_{\star}$. As shown in appendix A, this definition implies that $L=R_{E} \arccos \left(1-2 f_{A} / D\right)$, where $R_{E}$ is the radius of Earth, and $f_{A}=A /\left(4 \pi R_{E}^{2}\right)$ is the area fraction of $A$ relative to the surface of the entire sphere.

As an example with anticorrelations (and nonstationary correlation function), consider the idealized case of a spatial domain $\mathcal{S}$ of size $A=1$ that is divided into two subsections of size $a$ and $1-a$ (with $0 \leq a \leq 1$ ), and let all locations within either subsection have a correlation of $\rho(\mathbf{r}$, $\left.\mathbf{r}^{\prime}\right)=1$, and all cross correlations between the subsections be equal to $\rho\left(\mathbf{r}, \mathbf{r}^{\prime}\right)=r$ (with $\left.-1 \leq r \leq 1\right)$. Performing the two spatial averages involved in (1), or likewise (2), we obtain $\rho_{\star}=R_{\star}=a[a+(1-a) r]+(1-a)[(1-a)+a r]$. Simplifying and taking the reciprocal yields

$$
D=[1-2 a(1-a)(1-r)]^{-1} .
$$


For $r=0$ and $a=1 / 2$, this corresponds to the previous case with $D=N_{\text {box }}=2$. For $r<0$ and $0<a<1$, the correlation structure represents an idealized teleconnection dipole covering the domain $\mathcal{S}$. Setting $a=1 / 2$ yields $D=2 /(r+1)$, which shows that for negative $r$ the ESDOF measure grows beyond $D=2$, and for $r=-1$, corresponding to a "perfect teleconnection dipole" setting, it is not defined. Thus, $D$ does not behave like a conventional ESDOF measure in this case. In cases with perfectly anticorrelated subsections, $r=-1$, the measure is rather sensitive to the spatial partitioning $a$ of the teleconnection dipole, $D=[a-(1-a)]^{-2}$. Hence, whenever significant anticorrelations occur in the random field, then $D$ is not an ESDOF measure in the conventional sense, but a more general summarizing metric of the spatial correlation structure. The usefulness of such a metric, for applications to fields describing climate variability, is demonstrated in section 4.

Figure 3 illustrates a few of the abovementioned idealized cases with stationary variance, and how the corresponding value of $D$ comes about. Specifically, a case with a stationary Matérn class correlation function is shown in Fig. 3a, a case with a nonstationary (box-shaped) correlation function, but with the same value of $D$, is shown in Fig. 3b, and an example of an idealized teleconnection dipole in Fig. 3c.

\section{2) LOWER AND UPPER BOUNDS}

Both $D_{R}$ and $D_{\rho}$ attain their lowest possible values, denoted by $\min \left(D_{R}\right)$ and $\min \left(D_{\rho}\right)$, respectively (under variation of the spatial correlation function, given the spatial distribution of the local variance), if the variability is spatially coherent. Specifically,

$$
D_{R}=\min \left(D_{R}\right)=\overline{\sigma^{2}} / \bar{\sigma}^{2} \geq 1
$$

if $\rho\left(\mathbf{r}, \mathbf{r}^{\prime}\right)=1$ for all $\mathbf{r}, \mathbf{r}^{\prime}$ with $\sigma^{2}(\mathbf{r}), \sigma^{2}\left(\mathbf{r}^{\prime}\right)>0$, and

$$
D_{\rho}=\min \left(D_{\rho}\right)=1
$$

if $\rho\left(\mathbf{r}, \mathbf{r}^{\prime}\right)=1$ for all $\mathbf{r}, \mathbf{r}^{\prime}$. The lower bound of $D_{R}$ depends on the spatial distribution of $\sigma(\mathbf{r})$. For example, if $\sigma(\mathbf{r})=1$ over one hemisphere and zero over the other, then $\min \left(D_{R}\right)=(1 / 2) /$ $(1 / 4)=2$.

Corresponding upper bounds, on the other hand, do not exist. In cases with $\rho\left(\mathbf{r}, \mathbf{r}^{\prime}\right) \geq 0$ for all $\mathbf{r}, \mathbf{r}^{\prime}$, both measures may become arbitrarily large $\left(D_{\rho}, D_{R} \rightarrow \infty\right)$ for random fields approaching continuous spatial white noise. However, we may also have $D_{\rho}, D_{R} \rightarrow \infty$ in cases with nonzero decorrelation lengths if the random field exhibits anticorrelations, $\rho\left(\mathbf{r}, \mathbf{r}^{\prime}\right)<0$ for some $\mathbf{r}, \mathbf{r}^{\prime}$, that is, if there is strong cancelation between positive and negative fluctuations (e.g., a perfect teleconnection dipole). This latter case occurs, for instance, if the variability of the underlying field $X$ arises exclusively from redistribution within the spatial domain $\mathcal{S}$, such that the spatial average $\bar{X}$ is conserved (implying $\sigma_{0}^{2}=0$ ). For example, if the measures were applied to global surface pressure fields, then, because mass conservation implies $\sigma_{0}^{2}=0$, both measures are undefined. Hence, the ESDOF measures $D_{\rho}$ and $D_{R}$ are meaningful (and, thus, applicable) only if $\bar{X}$ is not conserved (implying $\sigma_{0}^{2} \neq 0$ ), for example, for global temperature or precipitation fields, or for regional fields of surface pressure. (a) Stationary $\rho\left(x, x^{\prime}\right)-$ Matérn class correlation functions

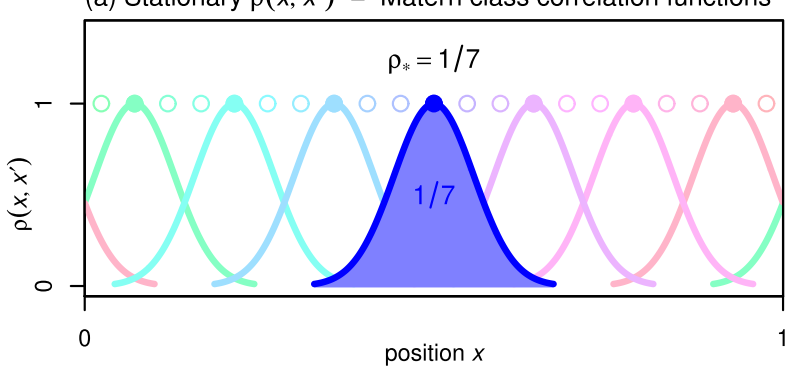

(b) Non-stationary $\rho\left(x, x^{\prime}\right)-$ Box correlation functions

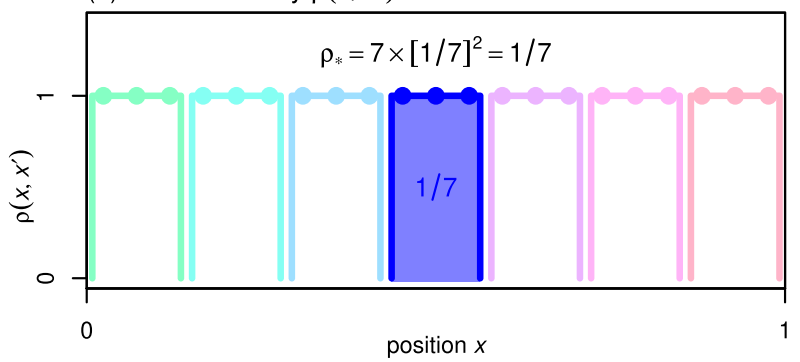

(c) Non-stationary $\rho\left(x, x^{\prime}\right)-$ Teleconnection dipole

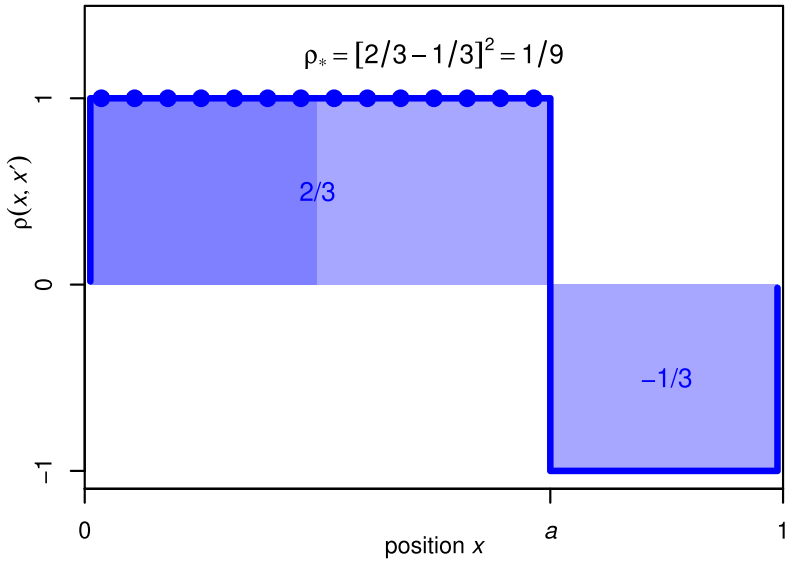

FIG. 3. Schematic illustration of different spatial correlation structures, shown, for simplicity, for the case of a one-dimensional, continuous, and cyclic spatial domain of size $A=1$. (a) Scenario with a Matérn class correlation function $\rho\left(x, x^{\prime}\right)$ that is stationary in space; i.e., it is identical for any reference location $x$. Colored dots indicate 21 example reference locations, for every third of which the correlation function is shown by a line with the same color. For $x=1 / 2$ (blue symbols) the integral of $\rho\left(x, x^{\prime}\right)$ is illustrated by shading, and its value is specified as a number. This scenario has $D=7$ spatial degrees of freedom. (b) As in (a), but for a nonstationary scenario with seven equally sized and uncorrelated boxes with $D=7$. (c) Scenario of a spatial domain split into two anticorrelated sections, representing an idealized teleconnection dipole. It corresponds to the setting $a=2 / 3, r=-1$, with $D=9$ according to Eq. (10); with reference locations shown only for $x<a$.

\section{3) SENSitivity to SPATIAL REDistribution}

In general, however, the presence of a process associated with redistribution does not necessarily imply an (unbounded) increase of the ESDOF measures. In particular, when the process of redistribution is combined with a nonconservative 
process (in the sense that it does not conserve $\bar{X}$ ), the effect of the redistribution can be an increase or decrease of the ESDOF measures, because the redistribution does not affect $\sigma_{0}^{2}$, but it may increase or decrease $\overline{\sigma^{2}}$.

For illustration, consider a simple energy balance model (EBM) for global surface temperature fields (referred to hereafter as the North-EBM; see North et al. 2011; Rypdal et al. 2015). In this model, temperature variability is driven by a spatiotemporal white noise forcing $\eta$ (stationary in space and time, implying $D_{R}=D_{\rho}$ ), and is modified by local thermal damping with time scale $\tau$, and by horizontal diffusion with length scale $\lambda$. The temporal evolution of this system is determined by the equation

$$
\frac{\partial X}{\partial t}+\frac{X}{\tau}-\frac{\lambda^{2}}{\tau} \nabla_{s}^{2} X=\eta
$$

where $\nabla_{s}^{2}$ denotes the Laplace operator in spherical coordinates.

If the white noise forcing $\eta$ is truncated at a given wavenumber $N_{T}$ (to ensure that spatial averaging over the random fluctuations leaves a nonzero residual, such that $\sigma_{0}^{2} \neq 0$ ), and if diffusion is switched off $(\lambda=0)$, then the resulting field of $X$ is associated with finite $D_{R}$ and $D_{\rho}$ according to the finiteness of $N_{T}$. However, if diffusion is included $(\lambda>0)$, then the variance of the local variability, $\overline{\sigma^{2}}$, is reduced, whereas $\sigma_{0}^{2}$ remains unaffected because the diffusion only redistributes temperature in space. Thus, according to (4), $D_{R}$ (and, thus, $D_{\rho}$ ) is also reduced. If, on the other hand, diffusion is replaced by another process of redistribution that is stochastically independent of the forced variability, then $\sigma_{0}^{2}$ is again unaffected, but $\overline{\sigma^{2}}$ is increased, and so are $D_{R}$ and $D_{\rho}$ in this case.

\section{c. Estimation}

To obtain estimators of the above ESDOF measures, consider a set of realizations of a discrete random field $X_{i}=X\left(\mathbf{r}_{i}\right), i=1, \ldots, N$, that is given at a finite number, $N$, of positions $\mathbf{r}_{i}$, being a discrete subset of the spatial domain $\mathcal{S}$. In the following, we use the notation $\sigma_{i}^{2}=\sigma^{2}\left(\mathbf{r}_{i}\right)$ and $\rho_{i j}=\rho\left(\mathbf{r}_{i}, \mathbf{r}_{j}\right)$, and the spatial average of some discrete field $x_{i}$ is then given by the weighted sum $\tilde{x}=\sum_{i=1}^{N} w_{i} x_{i}$, where the spatial weights $w_{i}$ are normalized according to $\sum_{i=1}^{N} w_{i}=1$. Using hat notation for estimators, we can write the estimator of the measure $D_{R}$ as

$$
\hat{D}_{R}=\widetilde{\hat{\sigma}^{2}} / \hat{\sigma}_{0}^{2}
$$

where $\hat{\sigma}_{0}^{2}$ is the estimated variance of $\tilde{X}$, and $\hat{\sigma}_{i}^{2}$ and $\hat{\sigma}_{0}^{2}$ are variance estimators.

To characterize the properties of $\hat{D}_{R}$ in terms of its standard error, we consider its relative bias, $\beta_{R}-1$, where $\beta_{R}=\left\langle\hat{D}_{R} / D_{R}\right\rangle$, and its relative scatter, $\delta_{R}=\operatorname{Var}\left(\hat{D}_{R} / D_{R}\right)^{1 / 2}$. As shown in appendix $\mathrm{B}$, both the bias and scatter vanish if $\rho_{\star}=1$, that is, $\beta_{R}=1$ and $\delta_{R}=0$ if $D_{R}=\min \left(D_{R}\right)$. For the case of the opposite limit, that is, if $\rho_{\star} \rightarrow 0$ (such that $D_{R} \rightarrow$ $\infty)$, appendix B shows that $\beta_{R}$ and $\delta_{R}$ increase monotonically, but stay below an upper bound, given by

$$
\beta_{R}<\beta_{R, \max }=\lim _{D_{R} \rightarrow \infty} \beta_{R}=\kappa /(\kappa-2),
$$

$$
\delta_{R}<\delta_{R, \max }=\lim _{\substack{D_{R} \rightarrow \infty \\ D_{R} \leq N}} \delta_{R}=\beta_{R, \max }[2 /(\kappa-4)]^{1 / 2},
$$

where $\kappa(>4)$ denotes the degrees of freedom of the variance estimates. Here $\beta_{R, \max }$ is the expected value and $\delta_{R, \max }^{2}$ is the variance of a scaled-inv- $\chi^{2}(\kappa, 1)$ distribution. These upper bounds are based on the additional assumption that $X_{i} \sim \mathcal{N}\left(0, \sigma_{i}^{2}\right)$, with stationary $\sigma_{i}^{2}$. It can be shown that, according to the above upper bounds, the bias is always smaller than the scatter, specifically, that the ratio $\left(\beta_{R, \max }-1\right)^{2} / \delta_{R, \max }^{2}<1$ and that it attains a maximum value of $1 / 8$ at $\kappa=8$.

To estimate the measure $D \rho$ from data with nonstationary variance, one may use the standardized time series $\left(X_{\sigma}\right)_{i}=\left(X_{i}-\hat{\mu}_{i}\right) /\left(\hat{\sigma}_{i}^{2}\right)^{1 / 2}$, where $\hat{\mu}_{i}$ denotes the estimated mean of $X_{i}$, to define the estimator of $D_{\rho}$ as

$$
\hat{D}_{\rho}=1 / \hat{\sigma}_{0, \sigma}^{2}
$$

where $\hat{\sigma}_{0, \sigma}^{2}$ is the estimated variance of $\widetilde{X_{\sigma}}$. The derivation of analytic expressions for the bias and scatter of $\hat{D}_{\rho}$ is more intricate, but it is found, from numerical simulations (see appendix B), that the above upper bounds for $\hat{D}_{R}$, given by (15) and (16), apply reasonably well also to $\hat{D}_{\rho}$ as long as $D_{\rho} \leq N$.

Estimating the normalized average radial covariance function, $R_{\star}(\theta)$, directly from two- (or higher-) dimensional discrete fields is not possible, because it involves the averaging operator $\overline{(\cdot)^{(\theta)}}$ at constant $\theta$, whereas the discrete locations $\mathbf{r}_{i}$ are generally not aligned in circles around each other. However, this issue can be circumvented by transforming the spatial fields into wavenumber space. Specifically, for the case of discrete fields covering the entire sphere, we can decompose the discrete fields into their spherical harmonic components (with total wavenumber $n$ and zonal wavenumber $m$ ) and then estimate the variance $\sigma_{n m}^{2}$ of each spherical harmonic time series. By analogy with Rypdal et al. (2015) - see their Eq. (10) where the same is done for the case of the North-EBM-we can then write the estimator $\widehat{\overline{R(\theta, \mathbf{r})}}=\sum_{n=0}^{N_{T}}\left[\left(\sum_{m=-n}^{n} \hat{\sigma}_{n m}^{2}\right) \mathcal{P}_{n}(\theta)\right]$ in terms of the estimated spherical harmonic component variances $\hat{\sigma}_{n m}^{2}$. Here $\mathcal{P}_{n}(\theta)$ is the $n$ th-order Legendre polynomial ${ }^{3}$ and $N_{T}$ is the truncation wavenumber (with, for example, $N_{T}=31$ in the case of the TraCE-21ka paleoclimate model simulation). This approach shifts the discretization in the spatial dimension to a discretization in wavenumber space, and the spatial dimension appears now in the analytically given Legendre polynomials. With this we can write the estimator of the normalized average radial covariance function (for fields covering the entire sphere):

$$
\hat{R}_{\star}(\theta)=\widehat{R(\theta, \mathbf{r})} / \widetilde{\hat{\sigma}^{2}}
$$

Accordingly, we can express the estimator of the ESDOF measure $D_{R, \text { fit }}$ as

\footnotetext{
${ }^{3}$ The Legendre polynomials are an orthogonal system if expressed as $\mathcal{P}_{n}(\cos \theta)$. Here they are expressed as $\mathcal{P}_{n}(\theta)$ to facilitate interpretation in spherical coordinates. To recover their orthogonality, the $\mathcal{P}_{n}(\theta)$ have to be multiplied by the spherical area weighting factor $\sin \theta$.
} 


$$
\hat{D}_{R, \text { fit }}=1 / M\left[\hat{R}_{\star, \text { fit }}(\theta)\right]
$$

where $\hat{R}_{\star \text { fit }}(\theta)$ denotes a parametric correlation function fit to $\hat{R}_{\star}(\theta)$. We do not characterize the properties of $\hat{D}_{R, \text { fit }}$, in terms of its bias and scatter, because of their dependence on the specific fitting procedure, which can be done in numerous different ways.

\section{d. Frequency dependence}

\section{1) DeFINITION}

In the following, the above ESDOF measures are equipped with an explicit dependence on frequency. For this purpose, the variances $\sigma^{2}(\mathbf{r})$ and $\sigma_{0}^{2}$ are interpreted as temporal variances, and are decomposed into their frequency components, that is, they are effectively replaced by the corresponding power spectral densities, $S(\nu ; \mathbf{r})$ and $S_{0}(\nu)$, respectively (with frequency $\nu$ ), assuming the field is stationary in time. By analogy with the relation $\sigma_{0}^{2}=\overline{\bar{R}}$ [see the explanation of (4)], we can then write $S_{0}(\nu)=\overline{\bar{R}}(\nu)$, where $R\left(\nu ; \mathbf{r}, \mathbf{r}^{\prime}\right)=\operatorname{Re}\left[C\left(\nu ; \mathbf{r}, \mathbf{r}^{\prime}\right)\right]$ is the covariance contribution from frequency $\nu$, given by the real part of the cross-spectral density $C\left(\nu ; \mathbf{r}, \mathbf{r}^{\prime}\right)$. Accordingly, the spatial correlation function at frequency $\nu$ is given by $\rho\left(\nu ; \mathbf{r}, \mathbf{r}^{\prime}\right)=R\left(\nu ; \mathbf{r}, \mathbf{r}^{\prime}\right) /\left[S(\nu ; \mathbf{r}) S\left(\nu ; \mathbf{r}^{\prime}\right)\right]^{1 / 2}$. Note, that the maps in Fig. 1 are indeed based on this quantity, and the maps in Fig. 2 on $\bar{\rho}(\nu ; \mathbf{r})$ and $\bar{R}(\nu ; \mathbf{r}) / \bar{S}(\nu)$. From this we can define the frequency-dependent versions of the average spatial extent of the fluctuations, by analogy with (1) and (2), as $\rho_{\star}(\nu)$ and $R_{\star}(\nu)$, respectively.

Consequently, we can define the frequency-dependent version of $D_{R}$, by analogy with (4), as

$$
D_{R}(\nu)=\bar{S}(\nu) / S_{0}(\nu),
$$

and the frequency-dependent version of $D_{\rho}$, by analogy with (5), as

$$
D_{\rho}(\nu)=1 / \rho_{\star}(\nu) .
$$

If $S(\nu ; \mathbf{r})$ is stationary in space at $\nu$, then $D_{R}(\nu)=D_{\rho}(\nu)$ at that frequency. Note, that the frequency-independent measure $D_{R}$ can be recovered from the frequency-dependent version by integration, performed separately on the numerator and the denominator:

$$
D_{R}=\int_{-\infty}^{\infty} \bar{S}(\nu) d \nu / \int_{-\infty}^{\infty} S_{0}(\nu) d \nu
$$

Similarly, the measure $D_{R}$ valid for some limited frequency band can be obtained by integration over that frequency range. Note, however, that $D_{\rho}$ can similarly be recovered from $D_{\rho}(\nu)$ only if $X(\mathbf{r})$ is white noise (in time) at each location $\mathbf{r}$. By analogy with (11) and (12), the lower bounds of these measures are given by

$$
D_{R}(\nu)=\min \left[D_{R}(\nu)\right]=\bar{S}(\nu) /\left[\overline{S^{1 / 2}}(\nu)\right]^{2} \geq 1
$$

if $\rho\left(\nu ; \mathbf{r}, \mathbf{r}^{\prime}\right)=1$ for all $\mathbf{r}, \mathbf{r}^{\prime}$ with $S(\nu ; \mathbf{r}), S\left(\nu ; \mathbf{r}^{\prime}\right)>0$, and

$$
D_{\rho}(\nu)=\min \left[D_{\rho}(\nu)\right]=1
$$

if $\rho\left(\nu ; \mathbf{r}, \mathbf{r}^{\prime}\right)=1$ for all $\mathbf{r}, \mathbf{r}^{\prime}$. Finally, the frequency-dependent version of the normalized average radial covariance function can be defined, by analogy with (6), as

$$
R_{\star}(\nu ; \theta)=\overline{R(\nu ; \theta, \mathbf{r})} / \bar{S}(\nu),
$$

and the frequency-dependent version of the ESDOF measure $D_{R, \text { fit }}$, by analogy with (8), as

$$
D_{R, \mathrm{fit}}(\nu)=1 / M\left[R_{\star, \mathrm{fit}}(\nu ; \theta)\right] .
$$

\section{2) Estimation}

By analogy with (14), the estimator of $D_{R}(\nu)$ is given by

$$
\hat{D}_{R}(\nu)=\widetilde{\hat{S}}(\nu) / \hat{S}_{0}(\nu),
$$

where $\hat{S}_{0}(\nu)$ is the estimated power spectral density of $\tilde{X}$ at $\nu$. The relative bias and scatter of this estimator are identical to those of the frequency-independent version, given by (15) and (16), respectively, with the exception that $\kappa$ now denotes the degrees of freedom of the spectral density (rather than variance) estimates.

Likewise, by analogy with (17), the estimator of $D_{\rho}(\nu)$ is given by

$$
\hat{D}_{\rho}(\nu)=1 / \hat{S}_{(0, \sigma)_{\nu}}(\nu),
$$

where $\hat{S}_{(0, \sigma)_{\nu}}(\nu)$ is the estimated power spectral density at $\nu$ of the spatial average of the standardized $X_{i}$, but now standardized and averaged in the Fourier domain. Specifically, it is estimated using the standardized Fourier transforms $\mathcal{F}\left[X_{i}\right]\left(\nu_{k}\right) /\left[\hat{S}_{i}(\nu)\right]^{1 / 2}$ at those discrete frequencies $\nu_{k}$ that fall into the spectral smoothing window (centered at $\nu$ ) involved in the estimation of $\hat{S}_{i}(\nu)$. Note that estimating $D_{\rho}(\nu)$ directly using all the correlations $\rho_{i j}(\nu)$, that is, according to $\hat{D}_{\rho}(\nu)=\left[\sum_{i=1}^{N} w_{i}^{2}+2 \sum_{i=2}^{N} \sum_{j=1}^{i-1} w_{i} w_{j} \hat{\rho}_{i j}(\nu)\right]^{-1}$ is also possible, but is computationally much more expensive.

Finally, by analogy with (18), the estimator of $R_{\star}(\nu ; \theta)$ is given by (for fields covering the entire sphere)

$$
\hat{R}_{\star}(\nu ; \theta)=\widehat{R(\nu ; \theta, \mathbf{r})} / \widetilde{\hat{S}}(\nu),
$$

where the estimator $\widehat{\overline{R(\nu ; \theta, \mathbf{r})}}=\sum_{n=0}^{N_{T}}\left\{\left[\sum_{m=-n}^{n} \hat{S}_{n m}(\nu)\right] \mathcal{P}_{n}(\theta)\right\}$ is now expressed in terms of the estimated power spectral densities of the spherical harmonic time series $\hat{S}_{n m}(\nu)$. With this we can express the estimator of the ESDOF measure $D_{R, \text { fit }}(\nu)$, by analogy with (19), as

$$
\hat{D}_{R, \mathrm{fit}}(\nu)=1 / M\left[\hat{R}_{\star, \mathrm{fit}}(\nu ; \theta)\right],
$$

where $\hat{R}_{\star, \text { fit }}(\nu ; \theta)$ denotes a parametric correlation function fit to $\hat{R}_{\star}(\nu ; \theta)$.

\section{3) ILLUSTRATION}

The frequency-dependent ESDOF measures are illustrated for the case of the North-EBM (introduced at the end of 
section $3 b$ ), for which the true ESDOF spectrum can be derived. Specifically, by decomposing the temperature field $X$ into spherical harmonics (see North et al. 2011; Rypdal et al. 2015), we obtain an expression for $D_{R}$ of the North-EBM,

$$
D_{R(\mathrm{ebm})}(\nu)=1+\left[(2 \pi \nu \tau)^{2}+1\right] \sum_{n=1}^{N_{T}} \frac{2 n+1}{(2 \pi \nu \tau)^{2}+\left[\left(\lambda / R_{E}\right)^{2} n(n+1)+1\right]^{2}},
$$

with the total wavenumber index $n$ and the truncation wavenumber $N_{T}$. To obtain (31) from (20), note, that, for the case of the North-EBM, (i) the power spectral density of the local variability $\bar{S}(\nu)$ is given by Rypdal et al. (2015) [see their Eq. (12)] and (ii) the power spectral density of the globally averaged temperature $S_{0}(\nu)$ is given by the term with $n=0$ of the same expression.

The above ESDOF spectrum (31) is shown in Fig. 4 (solid black line), for the North-EBM scaled to the dimensions of a sphere with the radius of Earth, $R_{E}$, and with the damping time scale set to $\tau=10$ years and the diffusion length scale to $\lambda=$ $1.25 \times R_{E}$, and using a truncation wavenumber of $N_{T}=31$ (corresponding to T31 horizontal resolution). The ESDOF spectrum is shown for a frequency range from millennial to monthly time scales. It turns out that the ESDOFs are strongly decreasing, from a few hundred at monthly time scales to just above one ESDOF at multidecadal time scales, following a powerlaw scaling (i.e., $\sim \nu^{p}$ ) with exponent $p=1$, and remain constantly low at centennial and longer time scales according to a scaling exponent of $p=0$. The break between these scaling regimes is located at a time scale of $2 \pi \tau \approx 63$ years for the above parameter choice. In the undamped regime $\left[\nu>(2 \pi \tau)^{-1}\right]$, the frequency dependence of the ESDOFs is caused by the fact that structures with smaller spatial scale (i.e., spherical harmonics with larger $n$ ) are diffused away more quickly than larger-scale structures. Thus, at higher frequencies, there are more small-scale structures contributing to the total variance of the local variability, relative to the variance contribution from the global average (given by the spherical harmonic with $n=0$, which is not affected by diffusion at all). In the damped regime $\left[\nu<(2 \pi \tau)^{-1}\right]$, the ESDOFs are constant as $\tau$ represents the longest time scale of the system (such that in this regime, all spherical harmonics have the same frequency scaling with $p=0$ in accordance with the white noise forcing).

The expected biased estimate in the limit of large $D_{R}$ is given by $\left\langle\hat{D}_{R}(\nu)\right\rangle=\beta_{R, \max }(\kappa) D_{R(\mathrm{ebm})}(\nu)$, according to (15), and is shown in Fig. 4 by the central dashed black line. The expected scatter of the estimate, in the same limit, is indicated by the upper and lower 5\% quantiles (upper and lower dashed black lines) of a scaled-inv- $\chi^{2}\left[\kappa, D_{R(\mathrm{ebm})}(\nu)\right]$ distribution that has the variance $\delta_{R \text {,max }}^{2}(\kappa) D_{R(\mathrm{ebm})}^{2}(\nu)$, in accordance with (16). Bias and scatter are shown for $\kappa=18.9$ degrees of freedom, consistent with a modified Daniell spectral smoothing window with 11 weights, which we also used for $\hat{S}(\nu)$ and $\hat{S}_{0}(\nu)$ when estimating $\hat{D}_{R}(\nu)$ from a numerical simulation of the North-EBM. From this simulation, $\hat{D}_{R}(\nu)$ is estimated using 7000 years of monthly mean fields at T31 horizontal resolution, shown in Fig. 4 by the red line. Additionally, from an ensemble of 1000 numerical simulations, we estimated the mean and the upper and lower $5 \%$ quantiles at each frequency (green lines). This confirms and illustrates both the coincidence of the bias and scatter with their expected values in the limit of large $D_{R}$ (at high frequencies) and their waning in the opposite limit (at low frequencies).

\section{e. Summary}

A frequency-dependent ESDOF measure, denoted $D_{R}(\nu)$, has been introduced in this section that is based on the integral of the spatial covariance function. It is identical, in its frequency-independent version, to the ESDOF measure used by Smith et al. (1994) and Jones et al. (1997), but here its interpretation in the presence of anticorrelations has been clarified. It has also been emphasized that its lower bound, that is attained in the case of globally coherent fluctuations, may still be greater than one if the local variance varies in space. If this is the case, the measure is more sensitive to those regions with larger variance. This effect can be avoided by using a variant of the measure, denoted $D_{\rho}(\nu)$, which involves a standardization at each location and frequency, and the lower bound of which is always equal to one. For spatial fields contaminated by noise (e.g., measurement noise), however, standardization may lead to noise amplification in regions where the local variance of the underlying field is small, resulting in a biased ESDOF estimate. It has also been shown how the average radial covariance function can be estimated from gridded fields, and how this allows to construct alternative ESDOF measures based on other aspects of the spatial covariance function than its integral. The usefulness of these measures lies in the possibility to investigate ESDOF-frequency spectra from which the frequency scaling of the spatial covariance structure may be estimated and visualized. It has been demonstrated, by an application to the North-EBM, that this scaling behavior may point to certain underlying physical processes.

\section{Application to climate model temperature and precipitation fields}

This section demonstrates the applicability of the previously defined ESDOF measures and their usefulness to characterize the covariance structure of climate variability. The measures are applied to 7000 years of monthly mean 2-m temperature and precipitation fields, both obtained from the TraCE-21ka paleoclimate model simulation and processed as described in section 2 for temperature.

As shown in Fig. 5a, the ESDOF estimator $\hat{D}_{R}(\nu)$, computed from global temperature fields, exhibits an almost uniform power-law scaling with an exponent of roughly $p=1 / 2$ across the entire frequency range, that is, from monthly up to millennial time scales. This scaling behavior is interrupted only by a small drop at ENSO time scales between 2 and 5 years and by the annual cycle (including its higher harmonics). According to this measure, there are about 220 ESDOFs at monthly and about 


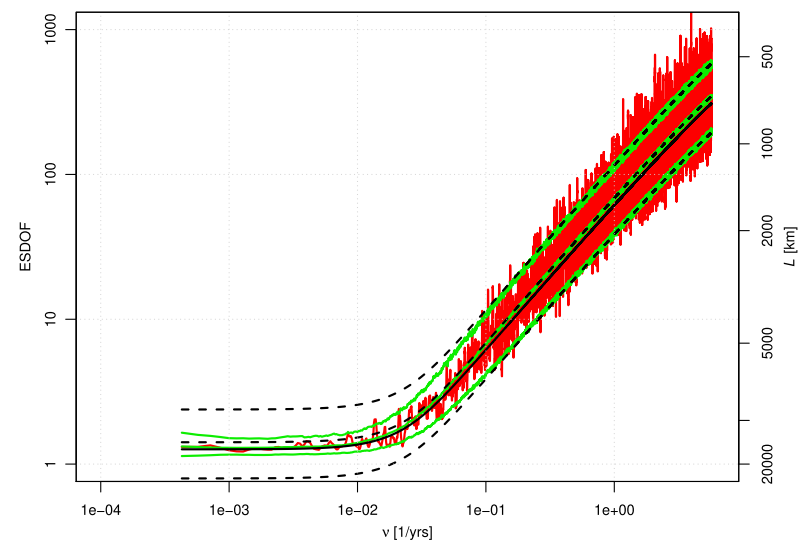

FIG. 4. ESDOF measure $D_{R}(\nu)$ (left vertical axis) and associated length scale $L$ (right vertical axis) as a function of frequency $\nu$, for the North-EBM. The true value $D_{R(\mathrm{ebm})}(\nu)$, according to Eq. (31), is shown by the solid black line, and the expected biased value, $\beta_{R} D_{R(\mathrm{ebm})}(\nu)$, by the central dashed black line. The upper and lower dashed black lines mark the upper and lower $5 \%$ quantiles of a scaled-inv- $\chi^{2}\left[\kappa, D_{R(\mathrm{ebm})}(\nu)\right]$ distribution, respectively. Indicated bias and scatter represent their expected upper bounds in the limit of large $D_{R}$. The red line shows $\hat{D}_{R}(\nu)$ estimated from a single numerical simulation of the NorthEBM. The green lines show the mean and the upper and lower 5\% quantiles estimated from an ensemble of 1000 numerical simulations.

four ESDOFs at millennial time scales. As explained in the previous section, this may indicate (i) a smaller spatial extent of the fluctuations or (ii) an increased occurrence of anticorrelations caused by teleconnections, toward the shorter time scales. As already suggested by Figs. 1 and 2, there seems to be a transition from scenario (i) at millennial to decadal time scales toward scenario (ii) at time scales shorter than decadal.

To make this more explicit, Fig. 5b shows estimates of the normalized average radial covariance function, $\hat{R}_{\star}(\nu ; \theta)$, for five different frequency bands. ${ }^{4}$ It turns out that from millennial to decadal time scales the $e$-folding length scale decreases [corresponding to scenario (i)] but then saturates toward the shorter time scales where changes in the spatial correlation structure are mainly associated with an increased occurrence of anticorrelations [corresponding to scenario (ii)]. This result can be expressed in terms of the ESDOF estimator $\hat{D}_{R, \mathrm{fit}}(\nu)$ by fitting an exponential correlation function $R_{\star}$,fit $(\nu ; \theta)=e^{-\theta / \theta_{e}(\nu)}$

\footnotetext{
${ }^{4}$ The normalized average radial covariance function for a frequency band bounded by $\nu_{1}$ and $\nu_{2}$ is obtained by integrating the numerator and denominator of (25) over that frequency interval. The five frequency bands are bounded by $\nu_{1}=(7000 \mathrm{yr})^{-1}, \nu_{2}=$ $(1000 \mathrm{yr})^{-1}$ for the millennial, by $\nu_{1}=(1000 \mathrm{yr})^{-1}, \nu_{2}=(100 \mathrm{yr})^{-1}$ for the centennial, by $\nu_{1}=(100 \mathrm{yr})^{-1}, \nu_{2}=(10 \mathrm{yr})^{-1}$ for the decadal, by $\nu_{1}=(10 \mathrm{yr})^{-1}, \nu_{2}=(14 \text { months })^{-1}$ for the annual, and by $\nu_{1}=(10 \text { months })^{-1}, \nu_{2}=(2 \text { months })^{-1}$ for the monthly band, where the annual cycle has been omitted. Narrow frequency bands (of 41 discrete frequencies) centered at the higher harmonics of the annual cycle have been omitted from the monthly band.

${ }^{5}$ Note that, given $\theta_{e}$, the rhs of (26) that involves an integral over $\theta$ (through the operator $M[\cdot]$ defined in section $3 a$ ) is written out explicitly by Jones et al. (1997) [see their Eq. (14)].
}

to $\hat{R}_{\star}(\nu ; \theta)$, such that both have the same $e$-folding length scale $\theta_{e}{ }^{5}$ The resulting values of $\hat{D}_{R, \text { fit }}(\nu)$ are shown in Fig. 5a (open dots). Whereas both ESDOF measures exhibit a similar increase from millennial up to decadal time scales, the two measures diverge at the higher frequencies. Specifically, whereas $\hat{D}_{R \text {,fit }}(\nu)$ saturates toward monthly time scales, $\hat{D}_{R}(\nu)$ continues to increase, with the largest discrepancy occurring within the monthly frequency band $\left[\hat{D}_{R \text {,fit }}(\nu)=45\right.$ vs $\left.\hat{D}_{R}(\nu)=183\right]$. If, alternatively, the $e$-folding length scale $\theta_{e}$ used to compute $\hat{D}_{R \text { fit }}(\nu)$ is obtained by fitting the exponential correlation function $R_{\star \text {,fit }}(\nu ; \theta)$ to $\hat{R}_{\star}(\nu ; \theta)$ over the entire interval $[0, \pi]$ [similar to the procedure of Jones et al. (1997)], $\hat{D}_{R \text {,fit }}(\nu)$ is getting closer to $\hat{D}_{R}(\nu)$ at all time scales, but the saturation toward the higher frequencies remains, as does the large discrepancy at monthly time scales (closed dots). These results explicitly demonstrate that at decadal and longer time scales the ESDOF measure $D_{R}(\nu)$, when applied to global 2-m temperature fields, essentially characterizes the spatial extent of the fluctuations, and is not notably affected by its specific sensitivity to anticorrelations (discussed in section 3b). Furthermore, it is an interesting result that the scaling behavior of the ESDOF measure $D_{R}(\nu)$ seems to be insensitive to the transition (at decadal time scales) from scenario (i) to scenario (ii), at least in this model simulation, as is reflected by the uniform scaling behavior across all frequencies.

In Fig. 6 a the estimator $\hat{D}_{R}(\nu)$ is compared to $\hat{D}_{\rho}(\nu)$, which exhibits a similar scaling behavior but values are smaller by roughly a factor of 1.5 , as a result of the standardization at each grid point and frequency. Here and for the following results we applied an additional smoothing in log-frequency before plotting the ESDOF spectra, separately to the numerator and the denominator of the measures, to increase the legibility of the figures. Since $D_{R}(\nu)$ is a frequency-dependent version of the second ESDOF measure of Jones et al. (1997), according to their Eq. (10), we can compare our results to the ESDOF values obtained by Jones et al. (1997) (see their Table 1). Estimating $\hat{D}_{R}$ from three different unforced model simulations, they obtain ESDOF values in the range from 20 to 83 (depending on the model) when using annual means, and from 10 to 39 when using decadal means. To compare this with our results, we have to integrate the numerator and the denominator of $D_{R}(\nu)$ up to $\nu=(1 \mathrm{yr})^{-1}$ or $\nu=(10 \mathrm{yr})^{-1}$, respectively, according to (22). This yields $\hat{D}_{R}=32$ for the annual and $\hat{D}_{R}=16$ for the decadal time scale, both of which lie within the above ranges obtained by Jones et al. (1997). Although TraCE-21ka is a forced model simulation, the forcing during our selected Holocene episode is relatively weak. By contrast, the observational ESDOF estimates of Jones et al. (1997), estimated from instrumental data for the episode from 1950 to 1990, are notably influenced by the anthropogenic warming signal, which explains the much smaller values of $\hat{D}_{R}=7$ for annual means and $\hat{D}_{R}=3$ for decadal means, because the forced response is expected to have a very large spatial scale, as discussed by Jones et al. (1997). Note that evaluation of $\hat{D}_{R}(\nu)$ from TraCE-21ka at the above two frequencies themselves (i.e., without integration) yields much larger values of $\hat{D}_{R}\left[\nu=(1 \mathrm{yr})^{-1}\right] \approx 100$ and $\hat{D}_{R}\left[\nu=(10 \mathrm{yr})^{-1}\right] \approx 50$, respectively, because in this case the slower variability, associated with fewer ESDOFs, is excluded. 
(a)

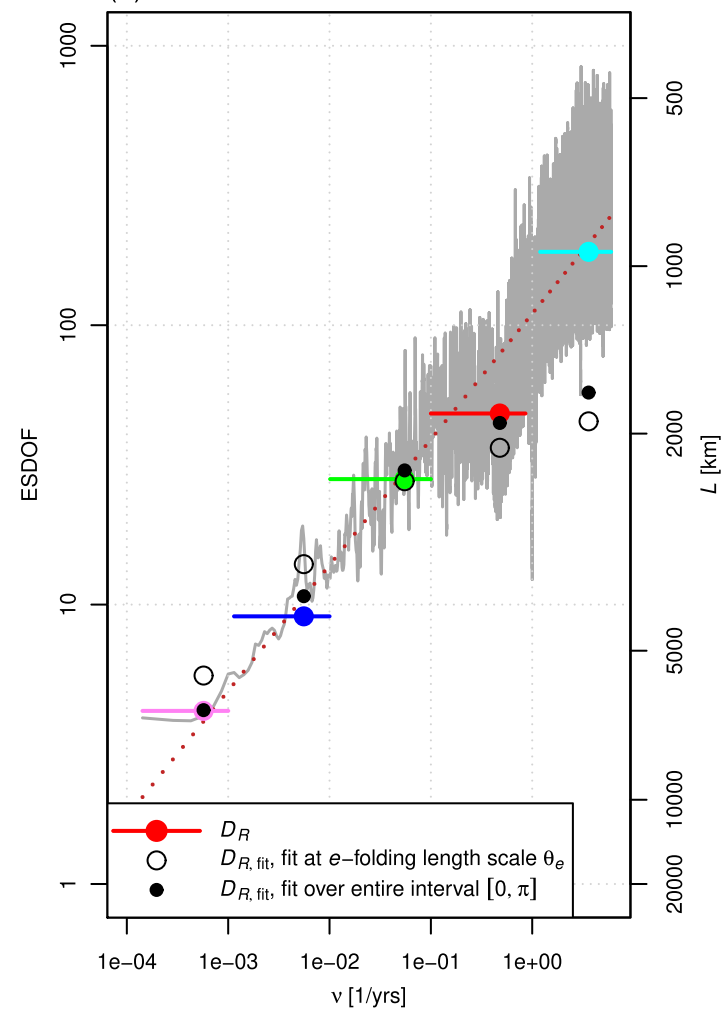

(b)

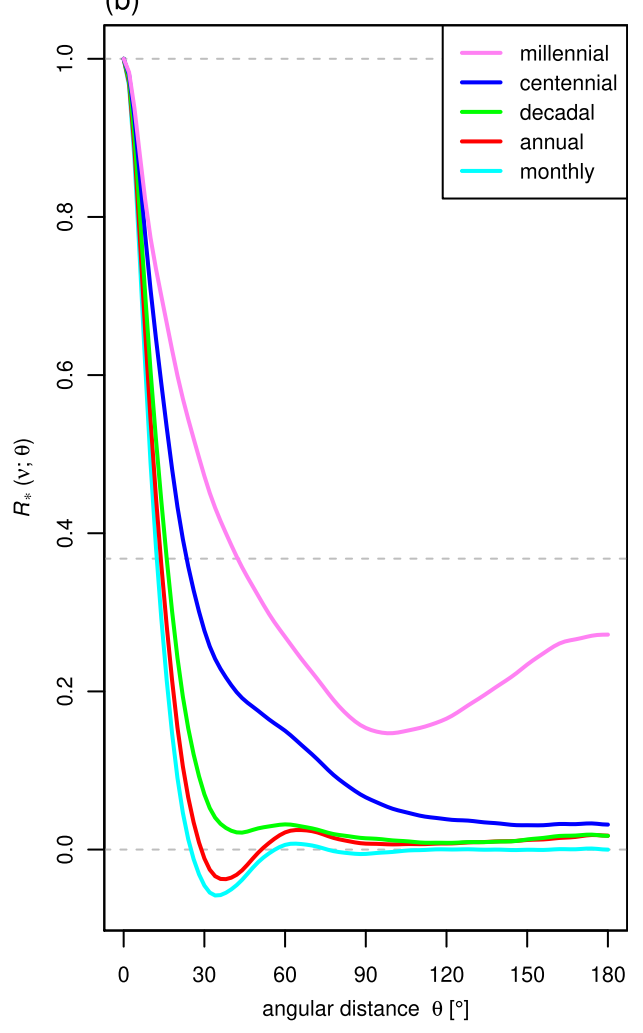

FIG. 5. (a) ESDOF estimator $\hat{D}_{R}(\nu)$ (left vertical axis) and associated length scale $L$ (right vertical axis) as a function of frequency $\nu$, estimated from global 2-m temperature fields of the TraCE-21ka paleoclimate model simulation, shown by the gray line. Colored line segments indicate the same measure, estimated from the millennial (violet), centennial (blue), decadal (green), annual (red), and monthly (cyan) frequency band, with colored dots at the central frequencies. Black dots indicate the ESDOF estimator $\hat{D}_{R, \text { fit }}(\nu)$ for the same frequency bands, based on an exponential correlation function $\hat{R}_{\star, \text { fit }}(\nu ; \theta)=e^{-\theta / \theta_{e}(\nu)}$ fit to $\hat{R}_{\star}(\nu ; \theta)$ either at the $e$-folding length scale $\theta_{e}$ (open dots) or over the entire interval $[0, \pi]$ (closed dots); see text for details. The dotted brown line follows a power-law frequency scaling with exponent $p=0.45$. (b) Estimator $\hat{R}_{\star}(\nu ; \theta)$, of the normalized average radial covariance function, for the same frequency bands, with colors as in (a). The central dashed gray line is at 1/e.

For all of the above estimates, the ESDOF values at decadal time scales are roughly half of the values at annual time scales. This suggests that the reduction of the global ESDOFs toward longer time scales is a robust feature across models and observations. This behavior is also consistent, at least qualitatively, with the simple North-EBM (see section 3d and Fig. 4), where the ESDOF reduction is caused solely by spatial diffusion. Although in TraCE-21ka the ESDOF scaling is flatter and it does not exhibit a scaling break, even such a scaling behavior can still be obtained by a simple diffusive EBM [the fractional EBM of Rypdal et al. (2015); not shown]. This suggests that diffusive processes might be the basic reason for the reduction of ESDOFs toward longer time scales, at least for internal (i.e., unforced) climate variability at time scales longer than those notably influenced by teleconnections. The above ESDOF spectra are characterizing the spatial covariance structure in a global mean sense. However, differences in this structure are to be expected between different regions, for example, between the extratropics (governed by quasigeostrophic scaling) and the tropics (characterized by strongly divergent circulations).
When the analysis is restricted to the tropics (defined here as the region between $30^{\circ} \mathrm{S}$ and $30^{\circ} \mathrm{N}$ ), shown in Fig. $6 \mathrm{~d}$, the scaling is different. Specifically, the scaling exponent $p$ is near zero at time scales longer than those of ENSO variability, with about 5 to 10 ESDOFs, depending on whether $\hat{D}_{R}(\nu)$ or $\hat{D}_{\rho}(\nu)$ is investigated, and a strong increase at shorter time scales up to about 80 ESDOFs at monthly time scales. In the tropics we also have $\hat{D}_{R}(\nu)>\hat{D}_{\rho}(\nu)$ across almost all frequencies. Note that the relation between the ESDOF measures (left vertical axis) and the length scale measure (right vertical axis) depends on the size $A$ of the spatial domain. Whereas for global fields, $D=1$ corresponds to $L=2000 \mathrm{~km}$ (the entire sphere), for the tropical region, for example, covering half of the sphere, $D=1$ corresponds to $L=10000 \mathrm{~km}$ (one hemisphere).

By contrast, in the northern extratropics, shown in Fig. 6b, a slight opposite scaling break is found near $\nu=(10 \mathrm{yr})^{-1}$. Thus, the frequency dependence of the ESDOFs is stronger at decadal to millennial and weaker at annual to monthly time scales. In addition, there is a narrow peak with fewer ESDOFs at $\nu=(13 \mathrm{yr})^{-1}$, which, however, is due to an unrealistic 
(a) T2m (global)

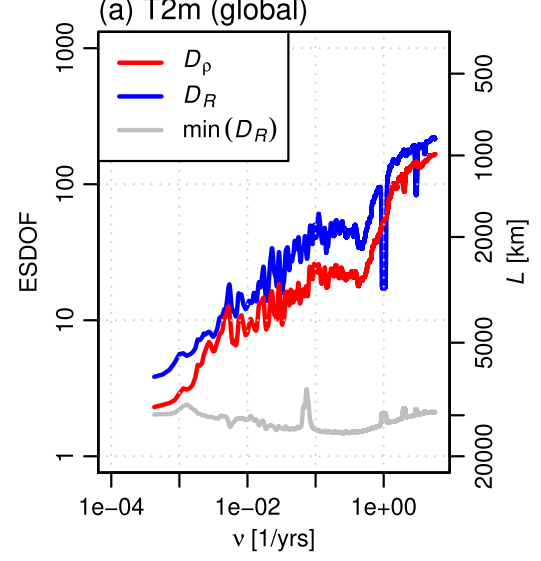

(d) T2m (tropics)

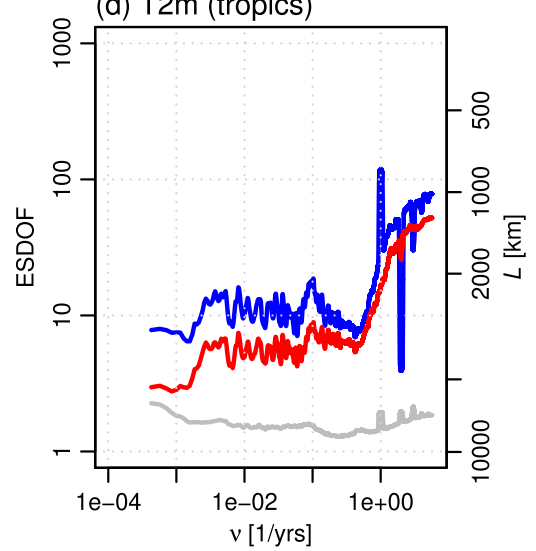

(b) $\mathrm{T} 2 \mathrm{~m}\left(>30^{\circ} \mathrm{N}\right)$

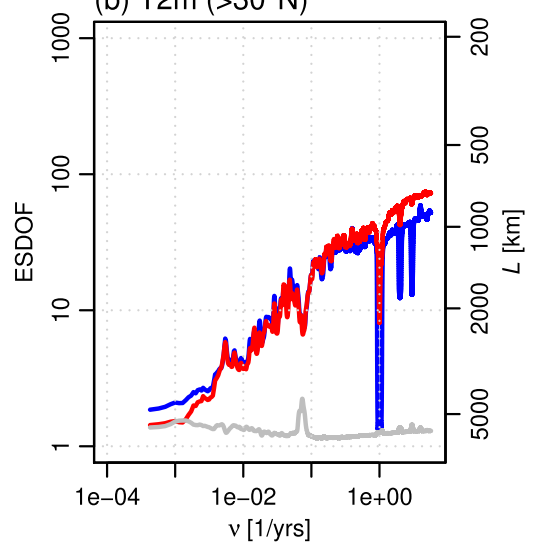

(e) $\mathrm{T} 2 \mathrm{~m}\left(>30^{\circ} \mathrm{N}\right.$, ocean)

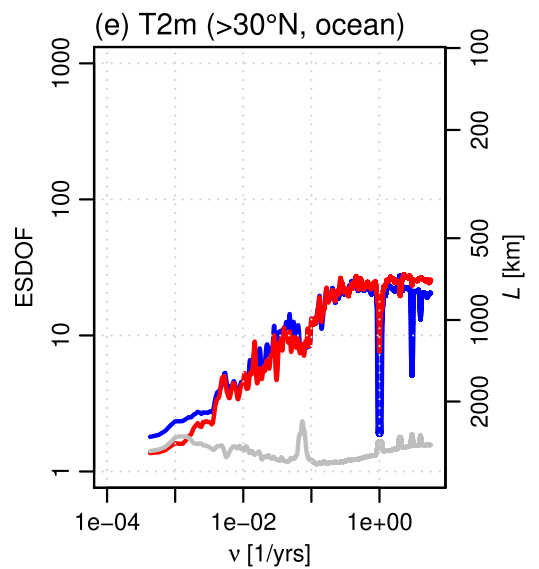

(c) Precip-strat (global)

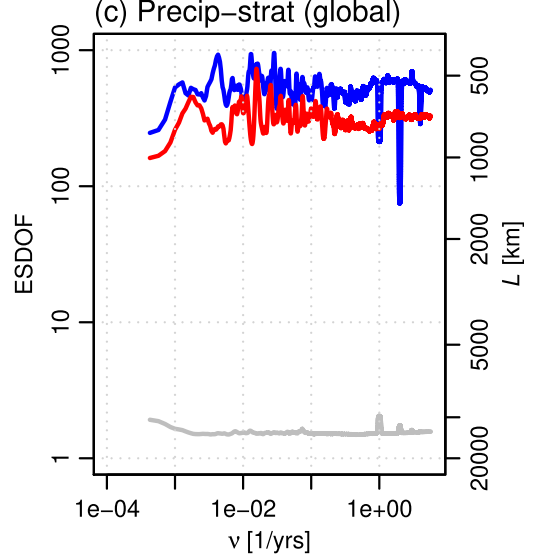

(f) Precip-strat (tropics)

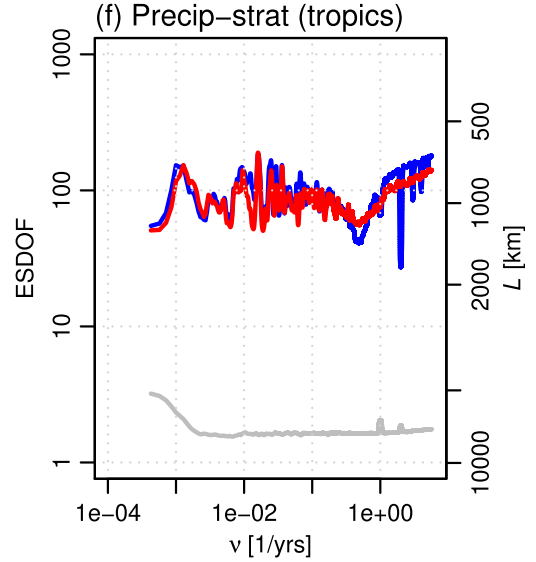

FIG. 6. ESDOF estimators $\hat{D}_{\rho}(\nu), \hat{D}_{R}(\nu)$, and the lower bound $\min \left[\hat{D}_{R}(\nu)\right]$ given the spatial distribution of $\hat{S}_{i}(\nu)$ at each frequency $($ left vertical axis) and associated length scale $L$ (right vertical axis) as a function of frequency $\nu$, with colors as indicated in (a), estimated from the TraCE-21ka paleoclimate model simulation. ESDOF estimates are shown for 2-m temperature (T2m) and for stratiform precipitation (Precip-strat), and for global fields (global), the region between $30^{\circ} \mathrm{S}$ and $30^{\circ} \mathrm{N}$ (tropics), the northern extratropics $\left(>30^{\circ} \mathrm{N}\right.$ ), and northern extratropical ocean areas $\left(>30^{\circ} \mathrm{N}\right.$, ocean $)$, as indicated above each panel.

feature of this model simulation, similar to what was found in other simulations with this model, although with different forcing and resolution, as reported by Danabasoglu (2008). This feature is associated with unrealistically large variability confined to the northern North Atlantic. It is this spatial confinement of the variance that causes the upward peak in the lower bound $\min \left[\hat{D}_{R}(\nu)\right]$ at the same frequency. The above scaling break is even more pronounced when the analysis is further restricted to northern extratropical ocean areas, shown in Fig. 6e. In that case, the scaling exponent is near zero at time scales shorter than 10 years, whereas at longer time scales the scaling is virtually unchanged. Note that at the annual

\footnotetext{
${ }^{6}$ Accordingly, $\hat{D}_{\rho}(\nu)$ should drop to a value of one. The reason why it does not in Figs. $6 \mathrm{~b}$ and $6 \mathrm{e}$ is the additional smoothing in logfrequency which we applied before plotting, affecting the annual cycle peak of the denominator of (28). By contrast, $\hat{D}_{R}(\nu)$ has annual cycle peaks in both the numerator and the denominator [see (27)], which are attenuated by the smoothing by the same factor, such that the measure itself is unaffected.
}

cycle frequency $\left[\nu=(1 \mathrm{yr})^{-1}\right]$ we obtain $\hat{D}_{R}(\nu)=\min \left[\hat{D}_{R}(\nu)\right]$ because there is only one coherent structure varying at this frequency (Figs. 6b,e). ${ }^{6}$ When the analysis includes both hemispheres, however, the annual cycle signature is more complex because of interhemispheric anticorrelations (Figs. 6a,d).

The frequency scaling of the ESDOF measures may be quite different when other variables are investigated. As an example, Fig. 6c shows $\hat{D}_{R}(\nu)$ and $\hat{D}_{\rho}(\nu)$ for global fields of stratiform (i.e., large scale) precipitation. These ESDOF spectra exhibit no scaling behavior at all, with a constant value of about 600 ESDOFs, in terms $\hat{D}_{R}(\nu)$, from monthly to millennial time scales. The reason for this different behavior is most likely the fact that stratiform precipitation is not a diffusive quantity, in contrast to temperature. For $\hat{D}_{\rho}(\nu)$ the value is smaller by roughly a factor of 2 , but again without any frequency dependence. A similar behavior is found for tropical stratiform precipitation, shown in Fig. 6f, except that both measures yield only about 100 ESDOFs and, additionally, exhibit a clear ENSO signature. When the ESDOF measures are applied to convective precipitation (not shown) a similar scaling to that of $2-\mathrm{m}$ temperature is 
found (although with much larger ESDOF values), probably because convection is closely related to the thermodynamics of the atmosphere and, thus, to temperature. By contrast, the processes leading to stratiform precipitation can be expected to exhibit spatial scales set by the large-scale fluid dynamics.

\section{Discussion}

As shown in section 3, the lower bound of the ESDOF measure $D_{R}$, defined by (4), depends on the spatial distribution of the variance [see (11)]. More specifically, the measure attains a value still greater than one, even if all locations within the spatial domain are perfectly correlated, which may seem to be counterintuitive, as one may expect $D_{R}=1$ in this case. This suggests defining the corrected measure $D_{R, c}=D_{R} / \min \left(D_{R}\right)=\bar{\sigma}^{2} / \sigma_{0}^{2}$, the lower bound of which is always one, irrespective of the variance distribution. Inspection of Figs. 6a, 6c, and $6 \mathrm{~d}$ indicates that the suggested correction would bring $D_{R}$ in close alignment with the measure $D_{\rho}$, which is independent of the distribution of variance. Given that the estimation of $D_{R, c}$ is computationally less expensive than estimation of $D_{\rho}$ (especially in the spectral domain, where standardization has to be performed at each frequency), one may prefer $D_{R, c}$ over $D_{\rho}$. However, Fig. $6 f$ shows that there are cases where the correction would rather create a mismatch with $D_{\rho}$. This implies that, in general, it is better to use $D_{R}$ together with its lower bound, or, alternatively, the measure $D_{\rho}$.

Nonetheless, it is useful to realize that, while $D_{R}$ represents a scale-oriented ESDOF measure, $D_{R, c}$ can be interpreted as a number-oriented measure. To understand this distinction, consider the idealized case of $N_{\text {box }}\left(=N_{\text {box }, 1}+N_{\text {box }, 0}\right)$ equally sized and uncorrelated boxes, with $N_{\text {box }, 1}$ boxes having variance one and $N_{\text {box }, 0}$ boxes having variance zero. Then, from the definition of the measures, we obtain $D_{R}=N_{\text {box }}$, which is the reciprocal of the (fractional) spatial scale of the box-shaped fluctuations, whereas we have $D_{R, c}=N_{\text {box }, 1}$, which is the number of the fluctuations.

Although, strictly speaking, this interpretation breaks down for smooth spatial variance distributions (as is the case for climate variability), it helps to differentiate our measure $D_{R}$ from the ESDOF measure $N_{\text {ef }}^{*}$ of Bretherton et al. (1999). Note, that for discrete spatial domains, we can rewrite our measures as $D_{R}=\left[\sum_{i=1}^{N} w_{i} \operatorname{Var}\left(X_{i}\right)\right] /\left[\sum_{i, j=1}^{N} w_{i} w_{j} \operatorname{Cov}\left(X_{i}, X_{j}\right)\right]$ and $D_{R, c}=\left[\sum_{i=1}^{N} w_{i} \operatorname{Var}\left(X_{i}\right)^{1 / 2}\right]^{2} /\left[\sum_{i, j=1}^{N} w_{i} w_{j} \operatorname{Cov}\left(X_{i}, X_{j}\right)\right], \quad$ respectively. Then, by replacing the raw anomalies $X_{i}$ by the squared anomalies $X_{i}^{2}$ (and, additionally, assuming that the $X_{i}$ are normally distributed), we obtain $D_{R}^{(2)}=\left[\sum_{i=1}^{N} w_{i} \operatorname{Var}\left(X_{i}\right)^{2}\right] /$ $\left[\sum_{i, j=1}^{N} w_{i} w_{j} \operatorname{Cov}\left(X_{i}, X_{j}\right)^{2}\right] \quad$ and $\quad D_{R, c}^{(2)}=\left[\sum_{i=1}^{N} w_{i} \operatorname{Var}\left(X_{i}\right)\right]^{2} /$ $\left[\sum_{i, j=1}^{N} w_{i} w_{j} \operatorname{Cov}\left(X_{i}, X_{j}\right)^{2}\right]$, respectively. It turns out, that $D_{R, c}^{(2)}$ is identical to the gridpoint formulation of $N_{e f}^{*}$ of Bretherton et al. (1999), see their Eq. (5). Thus, whereas $D_{R}$ is a scale-oriented ESDOF measure based on raw anomalies, $N_{\text {ef }}^{*}$ is a numberoriented measure based on squared anomalies.

Using squared anomalies has the advantage that anticorrelations do not counterintuitively increase the ESDOFs, such that a perfect teleconnection dipole is associated with one ESDOF, rather than diverging ESDOFs $\left(D_{R} \rightarrow \infty\right.$, as in our case). Hence, one may want to define a frequencydependent version of $D_{R}^{(2)}$. However, squaring anomalies in the time domain is not possible, because this distorts the signal in the frequency domain. ${ }^{7}$ Although squaring in the Fourier domain or in the cross-spectral density domain does actually turn anticorrelations into positive correlations, it also turns phase lags into negative correlations, again leading to a counterintuitive increase of the resulting ESDOF measure. Alternatively, one may square the real parts of the cross-spectral densities, but this yields a strong positive bias of the variance of the global average and, thus, a strong negative bias of the ESDOF measure, as it also occurs for $N_{\mathrm{ef}}^{*}$. However, the suggested bias correction of Bretherton et al. (1999), based on the assumption $\kappa \gg D$, is not applicable to our case, where $\kappa$ is small as it represents the spectral degrees of freedom, such that we may easily have $\kappa<D$. Thus, it would be necessary to provide an analytically derived bias correction valid also for small $\kappa$.

\section{Conclusions}

A frequency-dependent ESDOF measure is introduced that can serve as a simple and easy to compute summarizing metric of the covariance structure of a time-varying field.

- The underlying ESDOF measure is based on the variance of the spatial average of an anomaly field. The measure exploits the fact that the variance of the spatial average depends on the spatial correlation, as for largely uncorrelated anomalies the spatial average leaves only a small residual, whereas the residual is large for a spatially coherent field.

- An interpretation of the measure is provided for two settings: (i) for fields dominated by positive correlations, the measure characterizes the average spatial extent of the fluctuations; and (ii) for fields dominated by anticorrelated structures (due to, for example, teleconnections), it characterizes the relative spatial partitioning of the associated patterns into the respective anticorrelated spatial subsections.

- Two variants of the underlying measure are defined: with and without standardization of anomalies, the latter variant being identical, in its frequency-independent version, to the ESDOF measure used by Smith et al. (1994) and Jones et al. (1997).

- Both measures are equipped with an explicit dependence on frequency by replacing variances by power spectral densities, which allows for displaying ESDOF spectra across frequencies.

- Estimators for both measures are provided, and their bias and scatter are characterized.

- It is shown how the average radial covariance function can be estimated from gridded fields to construct alternative frequency-dependent ESDOF measures based on

\footnotetext{
${ }^{7}$ Note that squaring a harmonic oscillation doubles its frequency, and further distortions occur for discrete time signals.
} 
other aspects of the spatial covariance function than its integral.

These frequency-dependent ESDOF estimators are applied to temperature and to precipitation fields obtained from a paleoclimate model simulation. From the results we can conclude the following:

- Our frequency-dependent ESDOF measures are successful at providing concise information on the covariance structure of climate variability and, in particular, at detecting distinct space-time scaling regimes in the frequency domain.

- For global near-surface temperature fields, the ESDOFs exhibit a distinct reduction from monthly to millennial time scales, consistent with (i) the results of Jones et al. (1997) for annual to decadal time scales, and with (ii) a simple diffusive EBM, suggesting that horizontal diffusion is a basic reason for the reduction of the ESDOFs toward longer time scales.

- For stratiform precipitation, as an example of a nondiffusive climate variable, the ESDOFs do not exhibit any distinct frequency scaling between monthly and millennial time scales.

Numerous potential applications exist for these frequencydependent ESDOF measures. For example, as a reasonable next step, ESDOF spectra may be estimated from observational data, as this may significantly contribute to our understanding of climate variability by characterizing its space-time structure across wide ranges of frequencies. A challenge in this context will be the treatment of data gaps in space and time, in particular, when using paleoclimate data obtained from proxy records. In this latter case, frequency-dependent ESDOF estimates may provide useful information regarding the representative spatial scale of local paleoclimate reconstructions and how that scale varies with time scale. Data assimilation approaches in paleoclimate modeling (e.g., Hakim et al. 2016) may profit from this type of information. Another possible application is the comparison of models with observations, in order to validate or improve either of them. Laepple and Huybers (2014) detected large discrepancies in sea surface temperatures at long time scales for local, but not for large-scale variability, which implies different covariance structures in models versus observations. ESDOF spectra may also be used to identify climate variables and frequency bands for which the ESDOFs are independent of frequency, as in such cases the variability may possibly be described by statistical space-time factorization (provided the spatial statistics are stationary across frequencies), implying simplifications for prediction (Lovejoy et al. 2018). Finally, our frequency-dependent ESDOF measures may help to quantify the impact of external forcings on the space-time structure of climate variability, as various types of forcing may reveal different characteristic ESDOF fingerprints.

Acknowledgments. This is a contribution to the SPACE ERC project; this project has received funding from the European Research Council (ERC) under the European Union's Horizon 2020 research and innovation program (Grant Agreement 716092). The work profited from discussions at the Climate Variability Across Scales (CVAS) working group of the Past Global Changes
(PAGES) program. We also acknowledge discussions with Igor Kröner on the properties of the estimators, and the helpful comments of three anonymous reviewers.

\section{APPENDIX A}

\section{Length Scale Measure $L$}

The total surface area of a sphere with radius $R$ is given by $A_{\text {tot }}=4 \pi R^{2}$, and the area of a spherical cap by $A_{\text {cap }}=$ $2 \pi R^{2}(1-\cos \theta)$, where $\theta$ is half the opening angle of the cap, as seen from the center of the sphere. Then from (5), and requiring that $A_{\text {cap }} / A=A_{\text {cap }} /\left(f_{A} A_{\text {tot }}\right)=\rho_{\star}$, according to the definition of $L$ in section $3 \mathrm{~b}$, we have

$$
D=\frac{f_{A} A_{\text {tot }}}{A_{\text {cap }}}=\frac{2 f_{A}}{1-\cos \theta},
$$

where $D$ denotes $D_{R}$ or $D_{\rho}$. The radius $L$ of the cap along its curved surface is then given by

$$
L=R \theta=R \arccos \left(1-2 f_{A} / D\right) .
$$

Note that in the limit of large $D$ (i.e., if $D \gg 2 f_{A}$ ), $L$ can be approximated by $L \approx 2 R\left(f_{A} / D\right)^{1 / 2}$.

\section{APPENDIX B}

\section{Bias and Scatter of ESDOF Estimators}

Analytic expressions for $\beta_{R}=\left\langle\hat{D}_{R} / D_{R}\right\rangle$ and $\delta_{R}=\operatorname{Var}\left(\hat{D}_{R} / D_{R}\right)^{1 / 2}$ in the limit of large $D_{R}$ are derived, for the case of a standard normally distributed random field with stationary variance and equal spatial weighting, that is, for $X_{i} \sim \mathcal{N}\left(0, \sigma_{i}^{2}=1\right)$ and $w_{i}=1 / N$ for all $i$, and with all possible pairs of locations having the same correlation $\rho_{i j}$ (for $i \neq j$ ). This latter assumption requires that $\rho_{\min }<\rho_{i j} \leq 1$, with $\rho_{\min }=-1 /(N-1)$. We obtain $D_{R}=N /\left[1+(N-1) \rho_{i j}\right]$ and, in particular, $D_{R}=1$ if $\rho_{i j}=1$ and $D_{R} \rightarrow \infty$ as $\rho_{i j} \rightarrow \rho_{\text {min }}$.

By defining $\mathscr{b}=N^{-1} \sum_{i=1}^{N} \hat{\sigma}_{i}^{2}$ and $\mathscr{B}^{-1}=\hat{\sigma}_{0}^{2} D_{R}$, and using (14), we can write

$$
\beta_{R}=\langle\mathscr{b} \mathscr{B}\rangle=\langle\mathscr{b}\rangle\langle\mathscr{B}\rangle+\operatorname{Cov}(\mathscr{A}, \mathscr{B}) .
$$

Standard normality implies that $\langle\mathscr{C}\rangle=\left\langle\mathscr{B}^{-1}\right\rangle=1$ and, since $\mathscr{B}^{-1}$ is a $\chi^{2}$ variable, that

$$
\langle\mathscr{B}\rangle=\kappa /(\kappa-2),
$$

which is the expected value of a scaled-inv- $\chi^{2}(\kappa, 1)$ distribution. To investigate the behavior of the covariance term in (B1) in the limit of large $D_{R}$, note, that $\operatorname{Cov}\left(\mathscr{C}, \mathscr{B}^{-1}\right)=$ $D_{R} \operatorname{Cov}\left(\hat{\sigma}_{i}^{2}, \hat{\sigma}_{0}^{2}\right)=(2 / \kappa) D_{R} \operatorname{Cov}\left(X_{i}, \tilde{X}\right)^{2}$. Since the spatial stationarity of $\sigma_{i}^{2}$ and $\rho_{i j}$ implies $D_{R}=\operatorname{Cov}\left(X_{i}, \tilde{X}\right)^{-1}$, we obtain $\operatorname{Cov}\left(\mathscr{C}, \mathscr{B}^{-1}\right)=2 /\left(\kappa D_{R}\right)$. Similarly, we can obtain

$$
\operatorname{Var}(\not))=2 /\left(\kappa D_{R}^{(2)}\right),
$$

where $D_{R}^{(2)}$ is the ESDOF measure based on squared anomalies $X_{i}^{2}$, discussed in section 5, and which, for the 

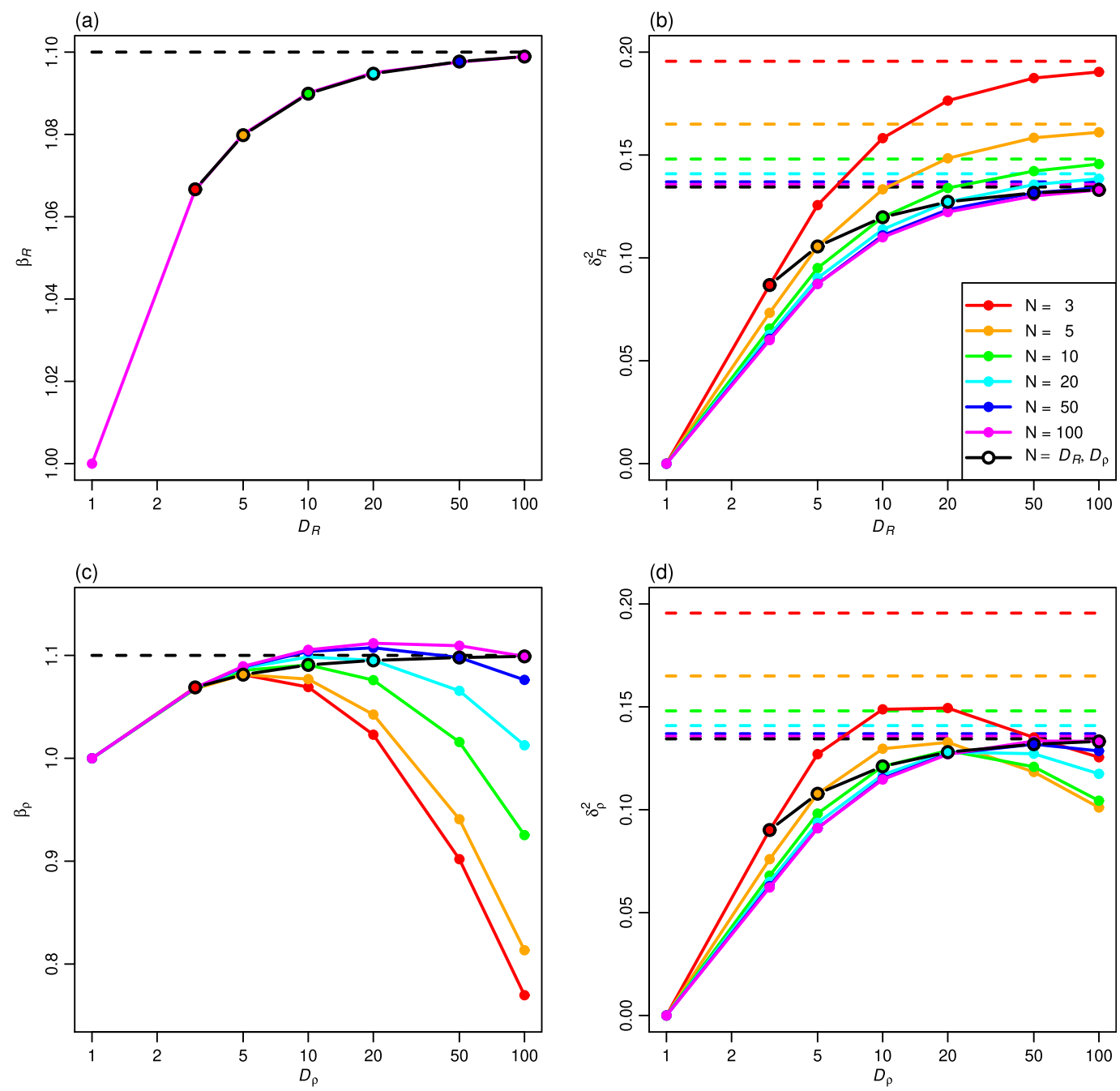

FIG. B1. Estimates of (a) $\beta_{R}$ and (b) $\delta_{R}^{2}$ as a function of $D_{R}$ and of (c) $\beta_{\rho}$ and (d) $\delta_{\rho}^{2}$ as a function of $D_{\rho}$ for different values of $N$ (solid colored lines and dots), obtained from numerical simulations according to the case described at the beginning of appendix B. Estimates from simulations with $N=D_{R}, D_{\rho}$ are connected by solid black lines with open dots. The dashed black line in (a) indicates the upper bound $\beta_{R \text {,max }}$, defined by (15), and in (b) the upper bound $\delta_{R \text {,max }}^{2}$, defined by (16). Dashed colored lines in (b) indicate the upper bounds $\delta_{R \text {,max }}^{2}(N)$, defined by (41). The same upper bounds (dashed lines) are repeated in (c) and (d) for comparison. In all cases we set $\kappa=22$.

present case, can be written as $D_{R}^{(2)}=N /\left[1+(N-1) \rho_{i j}^{2}\right]$. By noting that $\operatorname{Var}\left(\mathscr{B}^{-1}\right)=2 / \kappa$, we obtain the correlation $\operatorname{Corr}\left(\mathscr{C}, \mathscr{B}^{-1}\right)=\left(D_{R}^{(2)}\right)^{1 / 2} / D_{R}$. Using the expression for $D_{R}^{(2)}$, this can be expressed as $\operatorname{Corr}\left(\mathscr{b}, \mathscr{B}^{-1}\right)=[(N-1) /(N-d)]^{1 / 2}$, with $d=2 D_{R}-D_{R}^{2}$. For $D_{R}=1$, this yields a correlation of one. For increasing $D_{R}>1$, the correlation decreases monotonically, and approaches zero as $D_{R} \rightarrow \infty$. From this we imply that the covariance term in (B1) vanishes as $D_{R} \rightarrow \infty$. Hence, from (B2), $\beta_{R, \max }=\lim _{D_{R} \rightarrow \infty} \beta_{R}=\kappa /(\kappa-2)$. In the opposite limit, that is, if $D_{R}=1$, the numerator and the denominator of $\hat{D}_{R}$ are always identical, $\mathscr{C}=\mathscr{B}^{-1}$, such that the bias of the estimator vanishes, $\beta_{R}=1$. From a set of numerical simulations (see Fig. B1a) we infer that $\beta_{R}$ (solid black line) increases monotonically with $D_{R}$ and, thus, approaches its limit $\kappa /(\kappa-2)>1$ (dashed black line) from below. Hence, this limit represents an upper bound to $\beta_{R}$, as stated by (15) in section 3c.

Since the covariance term in (B1) vanishes in the limit of large $D_{R}$, we can write

$$
\begin{gathered}
\lim _{D_{R} \rightarrow \infty} \delta_{R}^{2}=\lim _{D_{R} \rightarrow \infty} \operatorname{Var}(\mathscr{C O B}) \\
=\lim _{D_{R} \rightarrow \infty}\left[\langle\mathscr{C}\rangle^{2} \operatorname{Var}(\mathscr{B})+\langle\mathscr{B}\rangle^{2} \operatorname{Var}(\mathscr{C})+\operatorname{Var}(\mathscr{C}) \operatorname{Var}(\mathscr{B})\right] .
\end{gathered}
$$

Because $D_{R} \rightarrow \infty$ implies $\rho_{i j} \rightarrow \rho_{\text {min }}$, we find from (B3), using the expression for $D_{R}^{(2)}$, that

$$
\lim _{D_{R} \rightarrow \infty} \operatorname{Var}(\mathscr{C b})=(2 / \kappa) /(N-1) .
$$


Additionally, because $\mathscr{B}^{-1}$ is a $\chi^{2}$ variable, we have

$$
\operatorname{Var}(\mathscr{B})=\beta_{R, \max }^{2}[2 /(\kappa-4)]
$$

which is the variance of a scaled-inv- $\chi^{2}(\kappa, 1)$ distribution. Then, using $\langle\mathscr{b}\rangle=1$, (B2), (B6), and (B7), we can rewrite (B5) as

$$
\lim _{D_{R} \rightarrow \infty} \delta_{R}^{2}=\delta_{R, \max }^{2}(N)=\beta_{R, \max }^{2} \frac{2}{(\kappa-4)}\left[1+\frac{1}{\beta_{R, \max }(N-1)}\right],
$$

which depends on $N$. In the opposite limit, that is, if $D_{R}=1$, the scatter vanishes, $\delta_{R}^{2}=0$, for the same reason as mentioned above for the bias. From the numerical simulations (see Fig. B1b), we infer that $\delta_{R}^{2}$ (solid colored lines) increases monotonically with $D_{R}$ and, thus, approaches its limit (B8) (dashed colored lines) from below, for any given $N$. Hence, the limit (B8) represents an upper bound to $\delta_{R}^{2}$ for that $N$. If, additionally, we let $N \rightarrow \infty$, then this limit reduces to $\lim _{N \rightarrow \infty} \lim _{D_{R} \rightarrow \infty} \delta_{R}^{2}=\delta_{R, \text { max }}^{2}=\beta_{R, \max }^{2}[2 /(\kappa-4)]$. Since $\lim _{N \rightarrow \infty} \lim _{D_{R} \rightarrow \infty} \delta_{R}^{2}=\lim _{D_{R} \rightarrow \infty} \delta_{R}^{2}\left(D_{R}=N\right)$, and because $\delta_{R}^{2}\left(D_{R}=N\right)$ (solid black line) increases monotonically with $D_{R}$, the limit $\delta_{R, \max }^{2}$ represents an upper bound to $\delta_{R}^{2}\left(D_{R}=N\right)$. As we can also infer from the numerical simulations that $\delta_{R}^{2}\left(D_{R} \leq N\right) \leq$ $\delta_{R}^{2}\left(D_{R}=N\right)$, the limit $\delta_{R \text {,max }}^{2}$ also represents an upper bound to $\delta_{R}^{2}$ for arbitrary $N$ as long as $D_{R} \leq N$, as stated by (16) in section $3 \mathrm{c}$.

For the estimator $\hat{D}_{\rho}$, the bias and the scatter can be similarly characterized by defining $\beta_{\rho}=\left\langle\hat{D}_{\rho} / D_{\rho}\right\rangle$ and $\delta_{\rho}=\operatorname{Var}\left(\hat{D}_{\rho} / D_{\rho}\right)^{1 / 2}$, respectively. Since an analytic treatment is more advanced in this case, we only compute $\beta_{\rho}$ and $\delta_{\rho}$ from the numerical simulations. Although the spatial stationarity of the simulated random fields implies $D_{R}=D_{\rho}$, it does not imply $\hat{D}_{R}=\hat{D}_{\rho}$. Nonetheless, for $D_{\rho}=N$ (solid black line in Fig. B1c), $\beta_{\rho}$ approaches the same upper bound $\beta_{R, \max }$ (dashed black line) as $D_{\rho} \rightarrow \infty$. For $D_{\rho}<N$, however, $\beta_{\rho}$ can slightly exceed $\beta_{R \text {,max }}$ for large values of $N$. Thus, the upper bound $\beta_{R \text {,max }}$ is only approximately applicable to $\hat{D}_{\rho}$. In cases with $D_{\rho}>N$, the behavior of $\hat{D}_{\rho}$ is very different from that of $\hat{D}_{R}$ and strong negative biases can occur $\left(\beta_{\rho}<1\right)$, in particular, for small values of $N$. These differences arise essentially from the fact that the correlation estimator, implicit to $\hat{D}_{\rho}$, is always biased toward zero. By contrast, $\delta_{\rho}^{2}$ is found to be almost identical to $\delta_{R}^{2}$ for both $D_{\rho}=N$ and $D_{\rho}<N$ (Fig. B1d), whereas for $D_{\rho}>N$, the scatter of $\hat{D}_{\rho}$ is much smaller than that of $\hat{D}_{R}$. From the above we conclude that the upper bounds $\beta_{R \text {,max }}$ and $\delta_{R \text {, max }}$ apply reasonably well also to $\hat{D}_{\rho}$ as long as $D_{\rho} \leq N$.

\section{REFERENCES}

Bretherton, C. S., M. Widmann, V. P. Dymnikov, J. M. Wallace, and I. Bladé, 1999: The effective number of spatial degrees of freedom of a time-varying field. J. Climate, 12, 1990-2009, https://doi.org/10.1175/1520-0442(1999)012<1990:TENOSD> 2.0.CO;2.

Cowtan, K., and R. G. Way, 2014: Coverage bias in the HadCRUT4 temperature series and its impact on recent temperature trends.
Quart. J. Roy. Meteor. Soc., 140, 1935-1944, https://doi.org/ 10.1002/qj.2297.

Danabasoglu, G., 2008: On multidecadal variability of the Atlantic meridional overturning circulation in the Community Climate System Model version 3. J. Climate, 21, 5524-5544, https:// doi.org/10.1175/2008JCLI2019.1.

Feldstein, S. B., and C. L. E. Franzke, 2017: Atmospheric teleconnection patterns. Nonlinear and Stochastic Climate Dynamics, C. L. E. Franzke, and T. J. O'Kane, Eds., Cambridge University Press, 54-104, https://doi.org/10.1017/ 9781316339251.004.

Hakim, G. J., J. Emile-Geay, E. J. Steig, D. Noone, D. M. Anderson, R. Tardif, N. Steiger, and W. A. Perkins, 2016: The last millennium climate reanalysis project: Framework and first results. J. Geophys. Res., 121, 6745-6764, https://doi.org/ 10.1002/2016JD024751.

He, F., 2011: Simulating transient climate evolution of the last deglaciation with CCSM3. Ph.D. thesis, University of WisconsinMadison, $171 \mathrm{pp}$.

Jones, P. D., T. J. Osborn, and K. R. Briffa, 1997: Estimating sampling errors in large-scale temperature averages. J. Climate, 10, 2548-2568, https://doi.org/10.1175/15200442(1997)010<2548:ESEILS>2.0.CO;2.

Laepple, T., and P. Huybers, 2014: Ocean surface temperature variability: Large model-data differences at decadal and longer periods. Proc. Natl. Acad. Sci., 111, 16 682-16 687, https:// doi.org/10.1073/pnas.1412077111.

Liu, Z., and Coauthors, 2009: Transient simulation of last deglaciation with a new mechanism for Bølling-Aller $\varnothing \mathrm{d}$ warming. Science, 325, 310-314, https://doi.org/10.1126/ science.1171041.

Livezey, R. E., and W. Y. Chen, 1983: Statistical field significance and its determination by Monte Carlo techniques. Mon. Wea. Rev., 111, 46-59, https://doi.org/10.1175/15200493(1983) $111<0046$ :SFSAID > 2.0.CO;2.

Lovejoy, S., L. del Rio Amador, and R. Hébert, 2018: Harnessing butterflies: Theory and practice of the Stochastic Seasonal to Interannual Prediction System (StocSIPS). Nonlinear Advances in Geosciences, A. A. Tsonis, Ed., Springer, 305-355, https:// doi.org/10.1007/978-3-319-58895-7_17.

North, G. R., J. Wang, and M. G. Genton, 2011: Correlation models for temperature fields. J. Climate, 24, 5850-5862, https:// doi.org/10.1175/2011JCLI4199.1.

Rypdal, K., M. Rypdal, and H.-B. Fredriksen, 2015: Spatiotemporal long-range persistence in Earth's temperature field: Analysis of stochastic-diffusive energy balance models. J. Climate, 28, 83798395, https://doi.org/10.1175/JCLI-D-15-0183.1.

Smith, T. M., R. W. Reynolds, and C. F. Ropelewski, 1994: Optimal averaging of seasonal sea surface temperatures and associated confidence intervals (1860-1989). J. Climate, 7, 949-964, https://doi.org/10.1175/1520-0442(1994)007<0949:OAOSSS> 2.0.CO;2.

Wallace, J. M., and D. S. Gutzler, 1981: Teleconnections in the geopotential height field during the Northern Hemisphere winter. Mon. Wea. Rev., 109, 784-812, https://doi.org/10.1175/ 1520-0493(1981)109<0784:TITGHF>2.0.CO;2.

Wang, X., and S. S. Shen, 1999: Estimation of spatial degrees of freedom of a climate field. J. Climate, 12, 1280-1291, https://doi.org/10.1175/1520-0442(1999)012<1280:EOSDOF> 2.0.CO;2. 